\title{
Influence of the critical Fe atomic volume on the magnetism of Fe-rich metallic glasses evidenced by pressure-dependent measurements
}

\author{
L. F. Kiss, ${ }^{1, *}$ T. Kemény, ${ }^{1}$ J. Bednarčík, ${ }^{2}$ J. Gamcová, ${ }^{2,3}$ and H.-P. Liermann ${ }^{2}$ \\ ${ }^{1}$ Institute for Solid State Physics and Optics, Wigner Research Centre for Physics, HAS, H-1525 Budapest, P.O. Box 49, Hungary \\ ${ }^{2}$ Deutsches Elektronen Synchrotron (DESY), Notkestrasse 85, D-22603 Hamburg, Germany \\ ${ }^{3}$ Department of Solid State Physics, P.J. Šafárik University, Košice, Slovakia
}

(Received 25 November 2015; revised manuscript received 19 May 2016; published 20 June 2016)

\begin{abstract}
Despite the intensive studies for decades, it is still not well understood how qualitatively different magnetic behaviors can occur in a narrow composition range for the Fe-rich Fe-transition metal (TM) amorphous alloys. In this study of amorphous $\mathrm{Fe}_{100-x} \mathrm{Zr}_{x}(x=7,9,12)$ metallic glasses, normal ferromagnetism $(\mathrm{FM})$ is found at $12 \% \mathrm{Zr}$ where only the FM-paramagnetic (PM) transition is observed at the Curie temperature, $T_{C}$. In contrast, spin-glass (SG)-PM transition at a temperature, $T_{g}$, called SG temperature, is only observed at $7 \% \mathrm{Zr}$, while in the transient re-entrant composition range $(x=8-11)$, an SG-FM transition at a temperature, $T_{f}$, called spin-freezing temperature, is also observed at low temperature besides the normal FM-PM transition at $T_{C}$. In order to understand this unusual behavior, a detailed characterization of pressure (atomic volume), composition, and temperature dependence of the magnetic properties is coupled with high pressure synchrotron $\mathrm{x}$-ray diffraction determination of the pressure dependence of the atomic volume. The results on $\mathrm{Fe}_{100-x} \mathrm{Zr}_{x}(x=7,9,12)$ are compared to those obtained for the $\mathrm{FM} \mathrm{Co}{ }_{91} \mathrm{Zr}_{9}$ metallic glass not showing any kind of anomalous magnetic properties. It is confirmed that the unusual behavior is caused by a granularlike magnetic structure where weakly coupled magnetic clusters are embedded into a FM bulk matrix. Since the mechanism of the magnetization reversal was found to be of the curling type rather than homogeneous rotation, the energy barrier determining the blocking temperature of the clusters is calculated as $A R$, where $A$ is the exchange constant and $R$ is the cluster size, in contrast to the usual characterization of the energy barrier by $K V$ where $K$ is the anisotropy energy and $V$ is the cluster volume. The volume fraction of the FM part is a fast changing function of the bulk composition: Almost $100 \%$ FM fraction is found at $12 \%$ of $\mathrm{Zr}$ while no trace of real FM is observed at 7 at $\% \mathrm{Zr}$. The driving force of this surprising magnetic character is the atomic volume: The lower the $\mathrm{Zr}$ content, the higher is the fraction of Fe atoms with compressed atomic volume having low magnetic moment. The percolation of their network separates the clusters from the FM bulk. The complex magnetic behavior of the Fe-rich $\mathrm{Fe}-\mathrm{Zr}$ amorphous system at low temperatures can thus be interpreted with the only assumption of a cluster-size distribution and a weak coupling of the clusters to the FM matrix. The introduction of this coupling is able to explain the opposite pressure dependence of $T_{g}$ and $T_{f}$. The threshold atomic volume in the low magnetic moment regions is found to be comparable to the atomic volume characteristic to the low-spin limit of the face-centered-cubic Fe alloys. The extensive literature results on the anomalous magnetism for various Fe-rich Fe-TM amorphous alloys and especially for the Fe-rich Fe-Zr glassy system are also found to be in agreement with this granular magnetic behavior.
\end{abstract}

DOI: 10.1103/PhysRevB.93.214424

\section{INTRODUCTION}

The origin of the peculiar magnetic properties [1-5] of Fe-rich Fe-transition metal $(T M)(T M=\mathrm{Zr}$, Ti, Hf, Nd, $\mathrm{Sc}, \mathrm{La}, \mathrm{Ce})$ metallic glasses at low temperatures has been a challenge for decades. Perhaps the most studied of these glasses are the amorphous $\mathrm{Fe}-\mathrm{Zr}$ alloys because in a very narrow composition range $\left(\mathrm{Fe}_{100-x} \mathrm{Zr}_{x}, 7 \leqslant x \leqslant 12\right)$, three different types of magnetic behavior can be observed. (1) $\mathrm{Fe}_{88} \mathrm{Zr}_{12}$ shows a paramagnetic (PM) to FM transition at the Curie temperature $\left(T_{C}\right)$ and remains in the FM state down to $4.2 \mathrm{~K}$. (2) Between $x=8$ to 11 , the behavior changes to a re-entrant spin-glass (RSG) type: Decreasing the temperature, the PM to FM transition is followed by an FM to SG transition at the spin-freezing temperature $\left(T_{f}\right)$. (3) $\mathrm{Fe}_{93} \mathrm{Zr}_{7}$ transforms directly from the FM into the SG state at the $\mathrm{SG}$ temperature

\footnotetext{
*Corresponding author: kissl@szfki.hu; kiss.laszlo.ferenc@ wigner.mta.hu
}

$\left(T_{g}\right)$. Another magnetic anomaly in these systems is the very rapidly increasing magnetic hardness on cooling as well as on decreasing $\mathrm{Zr}$ content [4], as deduced from coercivity measurements. Also, irreversible magnetization changes are observed below $T_{f}$ and $T_{g}$.

Although several explanations for this complex magnetic behavior have already been proposed, no consensus has been reached. One group of the models assumes the simultaneous presence of FM and antiferromagnetic (AF) exchange interactions in the Fe-rich amorphous $\mathrm{Fe}-\mathrm{Zr}$ alloys, causing exchange frustration [6]. The reason for this assumption is that the $\mathrm{Fe}-\mathrm{Fe}$ separation in these alloys is known to fluctuate around a value close to a critical $\mathrm{Fe}-\mathrm{Fe}$ separation $\left(d_{\mathrm{Fe}}=2.55 \AA\right)$, where the direct exchange integral between the $\mathrm{Fe}$ atoms changes sign, strongly decreasing with decreasing $\mathrm{Fe}-\mathrm{Fe}$ separation [7-10]. In this so-called wandering-axis ferromagnet model, the existence of a collinear FM matrix is questioned [5,11], and it is assumed that the spin structure is locally FM with small variation in neighboring spin directions; however, the local FM axis changes direction over distances of the order of $25 \AA$. The 
transition at the spin-freezing temperature, $T_{f}$ (called here as $T_{x y}$ ), is imagined as a homogeneous phase transition to an asperomagnetic state in which the fluctuating transverse spin components freeze at $T_{x y}$ (transverse spin freezing) on cooling, similar to that in the theoretical model of Gabay and Toulouse [12].

In another group of models, a magnetic structure inhomogeneous on an atomic scale is assumed. Here, AF [2,13-15] or nonmagnetic [16] or FM [17] clusters embedded in the FM matrix are responsible for the magnetic anomalies. Two subgroups might even be recognized in this way of thinking. In the first one, the existence of AF exchange interactions is also postulated similarly to the above mentioned homogeneous model; however, here the AF interactions play a role in the separation of the clusters from the FM matrix. In the model of Saito et al. [13], it is assumed that the SG state below $T_{f}$ is due to the freezing of frustrated spin clusters of AF Fe spins distributed in an FM matrix. Kaul et al. suggest that the amorphous $\mathrm{Fe}_{100-x} \mathrm{Zr}_{x}$ ( $x=8$ to 10) alloys consist of an FM matrix (infinite cluster) plus finite FM clusters separated and magnetically isolated by frustration zones [17-20]. This picture is supported by bulk magnetic [17] and FM resonance (FMR) data [18] and is further strengthened by measuring the effect of isothermal annealing on the magnetic behavior of amorphous Fe-Zr alloys using the FMR technique [19,20].

In the other subgroup of the inhomogeneous models, the anomalous low-temperature magnetic properties of Fe-rich amorphous Fe-Zr alloys have nothing to do with spin freezing. In the model of Read et al. and Moyo [4,14-16], the main effort is put on, explaining quantitatively the sharp temperature and composition dependence of the magnetic hardness. In the early form of the model $[4,14,15]$, the presence of AF clusters dispersed in an FM matrix was assumed, and the magnetic hardness was attributed to pinning to these clusters, considered as nonmagnetic inclusions below their Néel temperatures. Thus, the coercivity will be proportional to the volume fraction of the AF clusters. In this model, the transition at $T_{f}$ is simply caused by the breakdown of the kink-point relation ( $\chi_{\text {int }}=M / H_{\text {int }} \gg 1 / N$, where $N$ is the demagnetization factor of the sample and $H_{\text {int }}$ is the internal magnetic field) due to the rapid decrease in $\chi_{\text {int }}$ with decreasing temperature, caused by the increasing magnetic hardness. In this picture, $T_{f}$ depends on $N$ (i.e., on the sample geometry), and, consequently, it does not reflect a real physical transition.

In a revised version of this model [16], the regions having greater Fe density than a critical value (Fe-rich clusters) are assumed to contain $\mathrm{Fe}$ atoms in low-spin (LS) state with a moment of about $0.5 \mu_{B}$. The remaining FM matrix having a smaller Fe density is assumed to contain Fe atoms in high-spin (HS) state with a moment of about $2.8 \mu_{B}$. The domain wall pinning is then assumed to be caused by the high-density Fe-rich clusters, which can be considered as nonmagnetic inclusions because of their low moments. In order to explain the temperature dependence of coercivity in the new version, a new concept had to be introduced: the lattice parameter increases with increasing temperature because of thermal expansion, decreasing the size of the Fe-rich clusters. Hydrogen doping of Fe-rich amorphous Fe-Zr alloys decreases very rapidly the coercive field, which are similarly explained by the introduced lattice distortions [21]. Both versions of the model result in an exponential increase of the coercivity, $H_{c}$, with decreasing temperature, which is related to the upper tail of the assumed Gaussian distribution for the Fe concentration [4].

Though several theoretical works [22-24] assume the existence of competing FM and AF interactions in amorphous $\mathrm{Fe}-\mathrm{Zr}$ systems, some experiments cast serious doubts on the existence of AF ordered moments in Fe-rich amorphous $\mathrm{Fe}-\mathrm{Zr}$ alloys [25,26]. There is, however, a common point in almost all of the models mentioned above: They assume that the average atomic distances between Fe atoms fluctuate around a critical Fe-Fe separation. Decreasing the distance between two $\mathrm{Fe}$ atoms across the critical $\mathrm{Fe}-\mathrm{Fe}$ separation reduces drastically the atomic magnetic moment of Fe. A recent paper [27] relates this critical Fe-Fe separation to the critical volume, $V_{\mathrm{fcc} *} \approx 11.7 \AA^{3}$, separating the so-called LS and HS state of face-centered-cubic (fcc) $\mathrm{Fe}$ and concludes that the average $\mathrm{Fe}$ atomic volume of Fe-rich Fe-Zr metallic glasses is fairly close to it. This conclusion is based on a thorough analysis of density measurements for various Fe-rich Fe-TM metallic glasses [28]. Based on a careful study [29] of the composition dependence of the hyperfine parameters of Fe-rich $\mathrm{Fe}-\mathrm{Zr}$ metallic glasses, the existence of two different Fe local environments is confirmed, and they are identified with the exclusively Fe coordinated compressed and with the partially $\mathrm{Zr}$ coordinated Fe atoms of average volume, respectively. Taking into account these experimental results, a cluster-glass model was proposed [30] for the $\mathrm{Fe}_{100-x} \mathrm{Zr}_{x}$ system $(7 \leqslant x \leqslant 12)$ in which $\mathrm{Fe}_{93} \mathrm{Zr}_{7}$ is assumed to be an assembly of magnetic clusters with a blocking temperature distribution. The magnetic clusters in the alloys with higher $\mathrm{Zr}$ content are assumed to be weakly coupled to an infinite cluster (FM matrix). Using this model, on the base of systematic magnetic viscosity measurements, a curling-type reversal mechanism was proposed [31] for the description of the temperature and composition dependence of coercivity. The magnetic inhomogeneities could be associated with regions containing $\mathrm{Fe}$ atoms in HS state, surrounded by regions with $\mathrm{Fe}$ atoms in $\mathrm{LS}$ state. Polarized neutron scattering measurements showed [32-34] that in $\mathrm{Fe}_{92} \mathrm{Zr}_{8}$ and $\mathrm{Fe}_{90} \mathrm{Zr}_{10}$, the vast majority of the magnetic moments are found in some form of noncollinear order, and the noncollinear magnetic structure consists of regions that are spatially correlated with additional randomly correlated noncollinear moments. This picture is compatible with the cluster-type models and some form of the wandering-axis FM model. Since the noncollinear moments are spatially correlated in the whole temperature range below room temperature (RT) with no drastic change in the randomly correlated noncollinear moments below and above $T_{x y}$, the transverse spin freezing at $T_{x y}$ is not reflected in the polarized neutron scattering results.

Some open questions still remain concerning the clusterglass model applied for the Fe-rich amorphous $\mathrm{Fe}-\mathrm{Zr}$ alloys. The temperature and composition dependence of coercivity could be unambiguously interpreted by a curling-type reversal mechanism [31]. It is not clear, however, what determines the blocking temperature $\left(T_{B}\right)$ in this model where $T_{B}$ corresponds to $T_{g}$ and $T_{f}$ observed in the experiment. The shape anisotropy of the magnetic clusters earlier thought to determine $T_{B}$ is less probable, and it is difficult to explain its composition 
dependence [31]. It is obvious that $T_{B}$ should be related to the curling-type reversal mechanism, and one of the aims of this paper is to establish this relation. The second aim is to probe one of the most important prerequisites of the model (and practically of all models mentioned above), i.e., the existence of a critical $\mathrm{Fe}$ atomic volume separating the LS state (for smaller volumes) and the HS state (for larger volumes) by pressure-dependent magnetic measurements.

There is an early work $[35,36]$ on the pressure dependence of $T_{C}$ for the amorphous $\mathrm{Fe}_{100-x} \mathrm{Zr}_{x}(x=7,10,12,15,20$, 25 , and 40) alloy system, analyzing the observed decreasing $T_{C}$ with increasing pressure. It focuses only on the discussion of the magnetic behavior of the FM matrix, concluding that this alloy system is magnetically inhomogeneous. These data were included into a more comprehensive high-pressure study of magnetism on amorphous Fe-based alloys [37]. Though the alloy at the critical concentration $\left(\mathrm{Fe}_{93} \mathrm{Zr}_{7}\right)$ was also investigated by Shirakawa et al. [35], its low-field magnetization as a function of temperature was misinterpreted as showing a $T_{C}$ (i.e., as being a ferromagnet). Using the same data, Moyo interprets the pressure dependence of coercivity for the Fe-rich $\mathrm{Fe}-\mathrm{Zr}$ glasses in the framework of his revised model [16], in which the volume of the Fe-rich clusters increases by applying pressure.

Besides applying hydrostatic pressure, doping of Fe-rich $\mathrm{Fe}-\mathrm{Zr}$ amorphous alloys with a third element is another method to indicate that changes of the magnetic properties (e.g., $T_{C}$ ) are connected to a dominant volume effect. Zamani et al. [38,39] and Moubah et al. [40,41] studied the effect of light ion implantation $(\mathrm{H}, \mathrm{He}, \mathrm{B}, \mathrm{C}$, and $\mathrm{N})$ on the Curie temperature of amorphous $\mathrm{Fe}_{93} \mathrm{Zr}_{7}$ films, together with extended $\mathrm{X}$-ray absorption fine structure (EXAFS) measurements. They observed that the increase of $T_{C}$ is proportional to the increase in the average $\mathrm{Fe}-\mathrm{Fe}$ distance, which allowed them to conclude that the dominant cause of the $T_{C}$ enhancement of amorphous $\mathrm{Fe}_{93} \mathrm{Zr}_{7}$ upon doping is a volume effect. Similar effects were found for amorphous $\mathrm{Fe}_{89-x} \mathrm{~B}_{x} \mathrm{Zr}_{11}(x=0-10)$ alloys prepared by melt spinning where the Curie temperature and RT saturation magnetization increase almost linearly with B addition [42]. Also, an enhancement of FM was observed in $\mathrm{Fe}$-rich amorphous $\mathrm{Fe}-\mathrm{Zr}$ ribbons by hydrogen absorption [43].

Similar anomalous magnetic behavior was observed at low temperatures under pressure for amorphous Fe-TM alloys where $T M=\mathrm{Ti}$ [44], Hf [45], Nd [46], Sc [47], La [48], and $\mathrm{Ce}$ [49]. The concentration range where the abovementioned three types of magnetic state exist (FM, RSG, SG) depends on the type of the transition metal.

As we will show in this paper, the pressure dependence of the magnetic properties for the Fe-rich Fe-Zr metallic glasses can be well explained by the curling-type model. In this model, the energy barrier determining the blocking temperature turns out to be proportional to $A R$, where $A$ is the exchange constant and $R$ is the cluster size, in contrast to the usual characterization of the energy barrier by $K V$, where $K$ is the anisotropy energy and $V$ is the cluster volume. While the anisotropy-type model can explain several experimental features, our interpretation offers a simpler and more comprehensive understanding of the experimental findings. The introduction of a weak coupling of the clusters to the FM matrix enables us to explain the experimentally found opposite pressure dependence of $T_{g}$ and $T_{f}$.
The paper is organized as follows. Section II briefly describes the details of the sample preparation and measurements. The experimental results will be presented in Sec. III. The energy barrier determining the blocking temperature in the curling model will be calculated in Sec. IV, and the obtained formulas and the experimental results will be discussed in Sec. V. Finally, the conclusion of the present study will be summarized in Sec. VI.

\section{EXPERIMENT}

Amorphous $\mathrm{Fe}_{100-x} \mathrm{Zr}_{x}(x=7,9,12)$ and $\mathrm{Co}_{91} \mathrm{Zr}_{9}$ alloys were prepared by melt spinning in vacuum $(x=7,12)$ and in $\mathrm{He}$ of 1 mbar pressure $\left(x=9, \mathrm{Co}_{91} \mathrm{Zr}_{9}\right)$ [50]. The amorphous nature of the ribbons with a cross section of $1 \times 0.013 \mathrm{~mm}^{2}$ was verified by Mössbauer spectroscopy. The magnetic properties were measured using the MPMS-5S superconducting interference device (SQUID) magnetometer up to $5 \mathrm{~T}(50 \mathrm{kOe})$ in the temperature range of $1.8 \mathrm{~K}$ $\leqslant T \leqslant 300 \mathrm{~K}$. The thermomagnetic curves were recorded for the samples showing any magnetic transitions below RT. First, the samples were cooled down from RT to $5 \mathrm{~K}$ in zero magnetic field and were measured in a field of 10 Oe with increasing temperature up to RT [zero field cooling (ZFC) mode]. Then, the samples were cooled down again from RT to $5 \mathrm{~K}$ in the measuring field of $10 \mathrm{Oe}$, and the measurement was repeated like before [field cooling (FC) mode]. Because of the remanent field of the superconducting magnet (about $-1 \mathrm{Oe}$ ), the cooling field in the ZFC mode is not exactly zero, causing the negative magnetization observed at the lowest temperatures in some curves.

Samples were compressed under hydrostatic pressure up to 1.2 GPa in a miniature piston-cylinder $\mathrm{CuBe}$ pressure cell [51] using a mineral oil as the pressure-transmitting medium, and the cell with the sample was put into the SQUID magnetometer. The pressure is measured by detecting the shift with pressure of the superconducting transition temperature of $\mathrm{Pb}$ (at around $7 \mathrm{~K}$ ) put in the pressure cell together with the sample. The labels in all figures in this paper refer to these pressures measured at low temperatures. With increasing temperature, the pressure increases due to the different thermal expansion of the cell and the pressure-transmitting medium. To determine the rate of change of the various transition temperatures exactly, the values of pressure at temperatures close to them were used ( $p_{\text {corr }}$ in some figures), correcting for the thermal expansion effect described above [51].

In situ high-pressure $\mathrm{x}$-ray diffraction (XRD) experiments were performed at the Extreme Condition Beamline P02.2 of PETRA III (Hamburg, Germany). The RT pressure dependence of compressibility of the as-prepared metallic glasses was continuously monitored using a diamond anvil cell (DAC). The principal diffuse peak was detected in transmission mode to determine the volume changes under high pressure. The energy of the synchrotron radiation was set to $42.85 \mathrm{keV}$, which corresponds to the wavelength of $\lambda=0.28934 \AA$. KirkpatrickBaez (KB) mirrors were used to focus the photon beam down to $2 \times 2 \mu \mathrm{m}^{2}$ [52]. Two-dimensional XRD patterns were collected using a fast flat panel detector XRD1621 from PerkinElmer $\left(2048\right.$ pixels $\times 2048$ pixels, $200 \times 200 \mu \mathrm{m}^{2}$ pixel size, intensity resolution of 16 bit) carefully mounted 
orthogonally to the x-ray beam. $\mathrm{CeO}_{2}$ standard from National Institute of Standards and Technology (NIST) was used to calibrate the sample-to-detector distance and tilt of the imaging plate relative to the beam path. Neon was used as a pressure transmitting medium inserted into the sample chamber using a gas loader from Sanchez Technology. The pressure acting on the sample was determined using a ruby fluorescence scale from Mao et al. [53]. The pressure was increased in small steps using a pressure membrane attached to the back of the DAC and controlled by a pressure controller from Sanchez Technology. Two-dimensional XRD patterns were integrated in $q$ space using the software package FIT2D [54].

Using in situ high-pressure XRD measurements, we determined the pressure dependence of the relative volume change $d V / V_{0}$ for $\mathrm{Fe}_{91} \mathrm{Zr}_{9}$ and $\mathrm{Co}_{91} \mathrm{Zr}_{9}$ glasses at ambient temperature up to pressures of $40 \mathrm{GPa}$. It should be noted here that compressibility data were obtained by tracing the position of the principal diffuse peak: $-\Delta V / V_{0}=-\left[\left(q_{0} / q\right)^{3}-1\right]$, where $q_{0}$ and $q$ are positions of the principal diffuse peak at a reference ( $p=0.1 \mathrm{GPa}$ ) and given pressure, $p$, respectively [55]. It is worth noting that the ratio between the position of the second and the first diffuse peak does not change with pressure and is almost the same for both alloys, i.e., 1.66. This may suggest that both alloys are compressed elastically. Bridgman [56] has presented the equation of state for compressed solids as follows: $-\Delta V / V_{0}=a_{1} p+a_{2} p^{2}+\ldots$, where $a_{1}(>0)$ and $a_{2}(<0)$ are constants. The bulk modulus at zero pressure $B_{0}=-V_{0}(d p / d V)_{p=0}$ can be calculated as $B_{0}=1 / a_{1}$, where $a_{1}=\kappa$ is called compressibility.

\section{RESULTS}

The thermomagnetic curves for $\mathrm{Fe}_{93} \mathrm{Zr}_{7}$ are shown in Fig. 1, measured at different hydrostatic pressures. The ZFC and FC curves are denoted by solid and open symbols, respectively. The inset shows the curves for the lowest two pressures $(0$ means atmospheric pressure $=0.0001 \mathrm{GPa}$; the other pressure

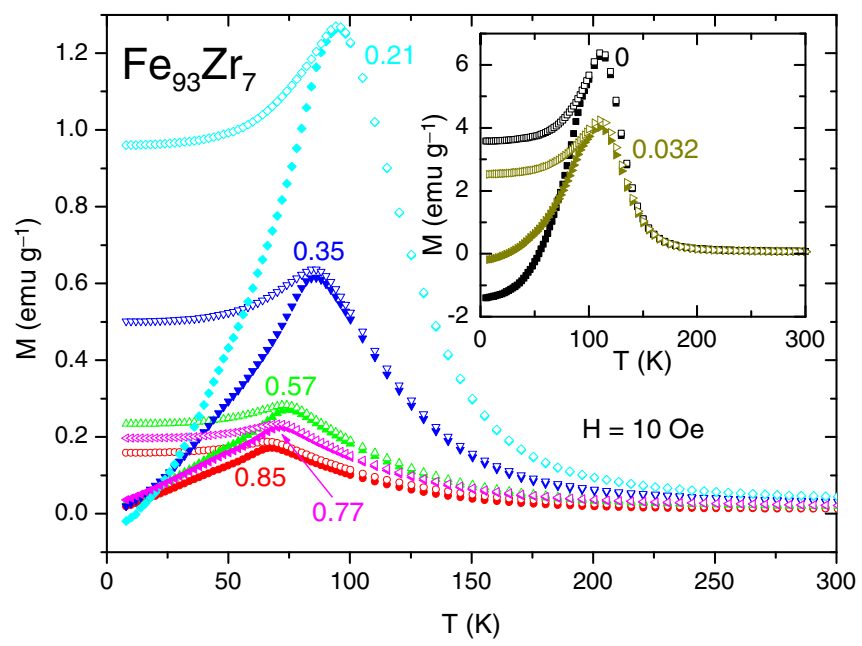

FIG. 1. Magnetization as a function of temperature for $\mathrm{Fe}_{93} \mathrm{Zr}_{7}$ measured after zero-field cooling (ZFC, solid symbols) and field cooling (FC, open symbols) in 10 Oe for different pressures. The pressure values are denoted as labels in GPa ( 0 means atmospheric pressure $=0.0001 \mathrm{GPa}$ ).

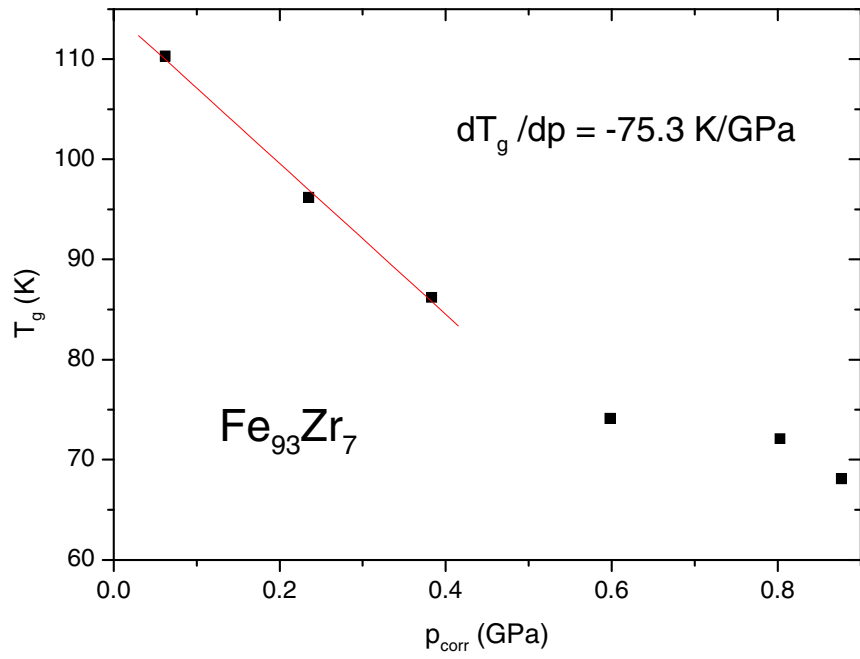

FIG. 2. Spin-glass temperature, $T_{g}$, as a function of pressure for $\mathrm{Fe}_{93} \mathrm{Zr}_{7} . T_{g}$ decreases with a rate of $-75.3 \mathrm{~K} \mathrm{GPa}^{-1}$ at lower pressures.

values are given in GPa as labels). Having a cusplike $M-T$ curve, this amorphous alloy behaves as a SG characterized by the temperature of the maximum called as SG temperature, $T_{g}$. The $T_{g}$ decreases with increasing pressure at a rate of $-75.3 \mathrm{~K} \mathrm{GPa}^{-1}$ up to about $0.4 \mathrm{GPa}$, tending to a saturation beyond this pressure, as displayed in Fig. 2 and listed in Table I. The low-field magnetization decreases substantially with increasing pressure. Similar pressure dependence of the low-field magnetization vs temperature curve was obtained by Shirakawa et al. [35] for a nominally same composition of the amorphous Fe-Zr system. However, they do not identify the cusp temperature as a SG temperature, $T_{g}$; instead, they interpret the decreasing magnetization with increasing temperature as an FM-PM transition with a Curie temperature, $T_{C}$. A similar decreasing trend of $T_{g}$ with increasing pressure was observed for amorphous Fe-La [48] and Fe-Ce [49] alloys. Figure 3 shows the field dependence of magnetization measured at $5 \mathrm{~K}$ at different pressures denoted as labels. Here it is also worth noting that the magnetization decreases remarkably with increasing pressure at any magnetic fields. Because of the significant high-field susceptibility found in all Fe-rich Fe-Zr alloys, real saturation of the magnetization cannot be achieved. The most simple and model-independent way is to take the magnetization value measured at $5 \mathrm{~T}(50 \mathrm{kOe})$ and $5 \mathrm{~K}$ as the measure of the saturation magnetization $\left(M_{5 \mathrm{~K}, 5 \mathrm{~T}}\right)$. Both $M_{5 \mathrm{~K}, 5 \mathrm{~T}}$ and $\ln M_{5 \mathrm{~K}, 5 \mathrm{~T}}$ decrease linearly with increasing pressure with the rate listed in Table I.

Pressure and temperature dependence of coercive field $\left(H_{c}\right)$ for $\mathrm{Fe}_{93} \mathrm{Zr}_{7}$ was measured between 10 and $30 \mathrm{~K}$ at selected pressures, as shown in Fig. $4 . H_{c}$ slightly increases with increasing pressure at $10 \mathrm{~K}$ with a saturating character. However, at higher temperatures, the pressure dependence is much weaker (see inset of Fig. 4). For both pressures, the coercivity decreases rapidly with temperature, as expected.

The existence of exchange anisotropy in these alloys is a delicate question since it is considered as a proof of the presence of AF interactions. Exchange anisotropy is claimed to exist in $\mathrm{Fe}_{92} \mathrm{Zr}_{8}$ [1]; however, a careful measurement for 
TABLE I. Pressure dependence of Curie temperature, $T_{C}$; saturation magnetization, $M_{5 \mathrm{~K} ; 5 \mathrm{~T}}, M_{0}$; and bulk modulus $B_{0}$. [Note: $T_{C}$ measured in $10 \mathrm{Oe}$ (see text), $M_{5 \mathrm{~K} ; 5 \mathrm{~T}}, M_{0}$ saturation magnetization: former measured at $5 \mathrm{~K}, 5 \mathrm{~T}$, latter determined from extrapolation to zero field (see text), $\kappa$ compressibility, $B_{0}$ bulk modulus at zero pressure, $\Gamma=d \ln M_{5 \mathrm{~K} ; 5 \mathrm{~T}} / d \ln V=-(1 / \kappa) d \ln M_{5 \mathrm{~K} ; 5 \mathrm{~T}} / d p$ Grüneisen parameter and $a_{2}$ coefficient of quadratic term in the Bridgman equation of state (see text).]

\begin{tabular}{|c|c|c|c|c|}
\hline & $\mathrm{Fe}_{93} \mathrm{Zr}_{7}$ & $\mathrm{Fe}_{91} \mathrm{Zr}_{9}$ & $\mathrm{Fe}_{88} \mathrm{Zr}_{12}$ & $\mathrm{Co}_{91} \mathrm{Zr}_{9}$ \\
\hline$T_{C}(\mathrm{~K})$ & - & $205 \pm 3^{a}$ & $261 \pm 3^{a}$ & $1200^{\mathrm{b}}$ \\
\hline$d T_{C} / d p\left(\mathrm{~K} \mathrm{GPa}^{-1}\right)$ & - & $-100 \pm 5$ & $-58.7 \pm 3.1$ & n.a. ${ }^{c}$ \\
\hline$d \ln T_{C} / d p\left(\mathrm{GPa}^{-1}\right)$ & - & $-0.60 \pm 0.05$ & $-0.26 \pm 0.02$ & n.a. ${ }^{\mathrm{c}}$ \\
\hline$T_{g}, T_{f}(K)$ & $110 \pm 3^{\mathrm{a}}$ & $16 \pm 1^{\mathrm{a}}$ & - & - \\
\hline$d T_{\mathrm{g}, \mathrm{f}} / d p\left(\mathrm{~K} \mathrm{GPa}^{-1}\right)$ & $-75.3 \pm 4.0$ & $45.5 \pm 0.4$ & - & - \\
\hline$d \ln T_{\mathrm{g}, \mathrm{f}} / d p\left(\mathrm{GPa}^{-1}\right)$ & $-0.769 \pm 0.015$ & $1.85 \pm 0.06$ & - & - \\
\hline$M_{5 \mathrm{~K}, 5 \mathrm{~T}}\left(\mathrm{emu} \mathrm{g}^{-1}\right)$ & $125.5 \pm 1^{\mathrm{a}}$ & $138.3 \pm 1^{\mathrm{a}}$ & $136.5 \pm 1^{\mathrm{a}}$ & $125.6 \pm 1^{\mathrm{a}}$ \\
\hline$M_{5 \mathrm{~K}, 5 \mathrm{~T}}\left(\mu_{B} / \mathrm{Fe}\right)$ & $1.41^{\mathrm{a}}$ & $1.61^{\mathrm{a}}$ & $1.67^{\mathrm{a}}$ & $1.53^{\mathrm{a}}$ \\
\hline$M_{0}\left(\mathrm{emu} \mathrm{g}^{-1}\right)$ & $103.4 \pm 1^{\mathrm{a}}$ & $127.9 \pm 1^{\mathrm{a}}$ & $132.9 \pm 1^{\mathrm{a}}$ & $125.6 \pm 1^{\mathrm{a}}$ \\
\hline$d \ln M_{5 \mathrm{~T}, 5 \mathrm{~K}} / d p\left(\mathrm{GPa}^{-1}\right)$ & $-0.49 \pm 0.03$ & $-0.34 \pm 0.01$ & $-0.23 \pm 0.01$ & $-0.0078 \pm 0.0007$ \\
\hline$\kappa\left(10^{-3} \mathrm{GPa}^{-1}\right)$ & n.a. ${ }^{\mathrm{c}}$ & $6.64 \pm 0.12$ & n.a. ${ }^{\mathrm{c}}$ & $6.57 \pm 0.08$ \\
\hline$B_{0}(\mathrm{GPa})$ & n.a. ${ }^{c}$ & $151 \pm 2.6$ & n.a. ${ }^{c}$ & $152 \pm 2$ \\
\hline$\Gamma$ & $74^{\mathrm{d}}$ & 51.1 & $34^{\mathrm{d}}$ & 1.18 \\
\hline$a_{2}\left(10^{-5} \mathrm{GPa}^{-2}\right)$ & n.a. ${ }^{\mathrm{c}}$ & $-6.26 \pm 0.46$ & n.a. ${ }^{c}$ & $-8.72 \pm 0.3$ \\
\hline
\end{tabular}

${ }^{\mathrm{a}} p=1$ bar.

${ }^{\mathrm{b}}$ Extrapolated from $M(T)$ curve since onset temperature of crystallization (Ref. [85]) $T_{x}$ is $T_{x}=800 \mathrm{~K}$; literature data for Co90 $\mathrm{Zr}_{10}: T_{C} \approx 998 \mathrm{~K}$ (Ref. [86]), 1615 K (Ref. [87]), and 1400 K (Ref. [61]).

${ }^{\mathrm{c}}$ Not available.

${ }^{\mathrm{d}}$ Calculated by the value of $\kappa$ measured for $\mathrm{Fe}_{91} \mathrm{Zr}_{9}$ (this work): $\kappa=6.6410^{-3} \mathrm{GPa}^{-1}$

$\mathrm{Fe}_{93} \mathrm{Zr}_{7}$ could not prove it [57]. The latter measurement was repeated in the present study since now the hysteresis loop could be measured at a lower temperature than before (at $1.8 \mathrm{~K}$ instead of $4.2 \mathrm{~K}$ ). In Fig. 5, the hysteresis loop is shown for $\mathrm{Fe}_{93} \mathrm{Zr}_{7}$ at $1.8 \mathrm{~K}$, first after cooling the sample in zero field, then after cooling in $5 \mathrm{~T}$. No significant shift of the hysteresis loop is observed upon FC, which does not support the existence of $\mathrm{AF}$ ordered moments in $\mathrm{Fe}_{93} \mathrm{Zr}_{7}$.

Figure 6 shows the ZFC and FC thermomagnetic curves for $\mathrm{Fe}_{91} \mathrm{Zr}_{9}$ measured at different hydrostatic pressures. This alloy behaves as a typical reentrant SG characterized with a spin-freezing temperature, $T_{f}$, defined here as the inflection

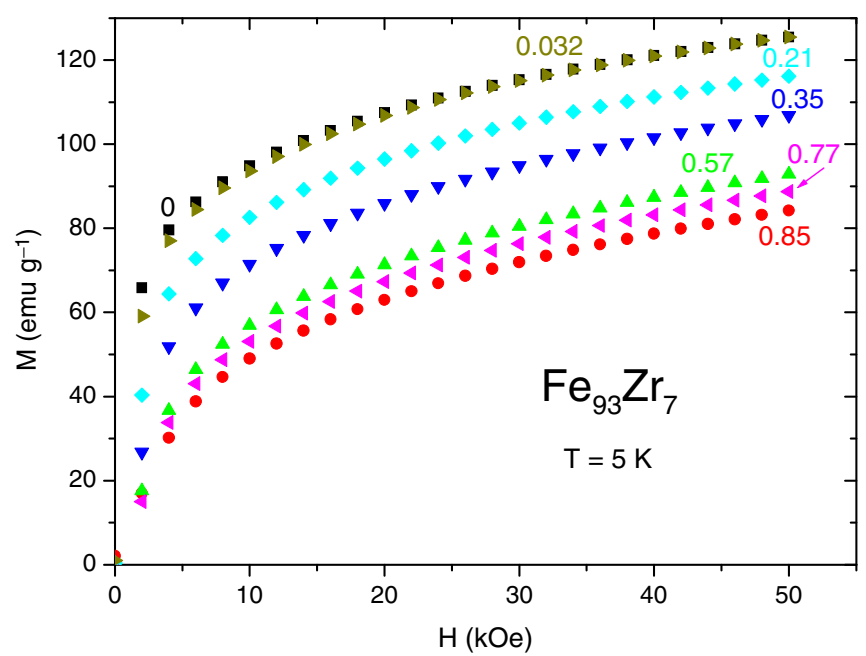

FIG. 3. Magnetization as a function of magnetic field for $\mathrm{Fe}_{93} \mathrm{Zr}_{7}$, measured at $5 \mathrm{~K}$ at different pressures denoted as labels in GPa $(0$ means atmospheric pressure $=0.0001 \mathrm{GPa}$ ). point of the initial increasing portion of the magnetization curve at low temperatures, and with a Curie temperature, $T_{C}$, defined similarly for the rapidly decreasing part of the curve. In contrast to the decrease of $T_{g}$ with increasing pressure observed for $\mathrm{Fe}_{93} \mathrm{Zr}_{7}, T_{f}$ rises with increasing pressure for $\mathrm{Fe}_{91} \mathrm{Zr}_{9}$ with an initial rate of $45.5 \mathrm{~K} \mathrm{GPa}^{-1}$ up to $0.4 \mathrm{GPa}$. An even larger rate can be observed beyond this pressure, as displayed in Fig. 7 and listed in Table I. $T_{C}$ decreases linearly with increasing pressure with a rate of $-100 \mathrm{~K} \mathrm{GPa}^{-1}$ (listed in Table I). For $\mathrm{Fe}_{91} \mathrm{Zr}_{9}$, a $d T_{C} / d p$ value of $-30 \mathrm{~K} \mathrm{GPa}^{-1}$ was reported from indirect Young's modulus measurements [58], while for $\mathrm{Fe}_{90} \mathrm{Zr}_{10}$, values of -38 [59-61], -60 [35], and -64

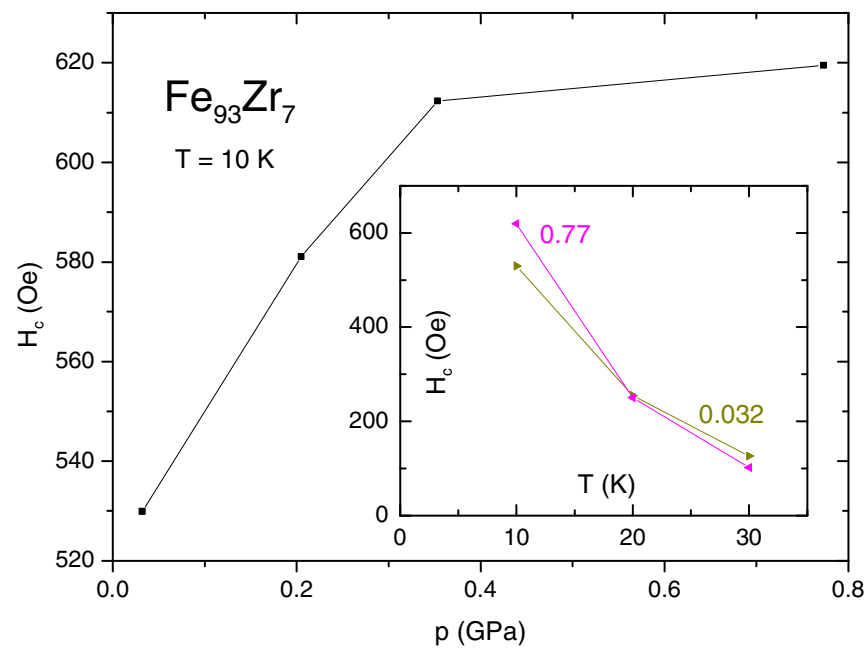

FIG. 4. Coercive field as a function of pressure for $\mathrm{Fe}_{93} \mathrm{Zr}_{7}$, measured at $10 \mathrm{~K}$. Inset: Coercive field as a function of temperature for $\mathrm{Fe}_{93} \mathrm{Zr}_{7}$ measured at different pressures denoted as labels in $\mathrm{GPa}$. 
[62] $\mathrm{K} / \mathrm{GPa}$ were deduced from the observed change in $T_{C}$ when pressure is applied. A $d \ln T_{C} / d p$ value of $-0.15 \mathrm{GPa}^{-1}$ was determined for $\mathrm{Fe}_{91} \mathrm{Zr}_{9}$ indirectly from forced-volume magnetostriction measurements [63]. The increase of $T_{f}$ and the decrease of $T_{C}$ with increasing pressure shift the shape of the thermomagnetic curve of $\mathrm{Fe}_{91} \mathrm{Zr}_{9}$ in the direction of the composition $\mathrm{Fe}_{92} \mathrm{Zr}_{8}$, i.e., as if the $\mathrm{Zr}$ content were decreased. The low-field magnetization decreases with increasing pressure. A similar trend of increasing $T_{f}$ and decreasing $T_{C}$ with increasing pressure was observed for amorphous Fe-La [48] and Fe-Ce [49] alloys. The field dependence of magnetization measured at $5 \mathrm{~K}$ is shown in Fig. 8 at different pressures. The magnetization decreases remarkably (but at a lesser rate than for $\mathrm{Fe}_{93} \mathrm{Zr}_{7}$ ) with increasing pressure at any magnetic fields. Both $M_{5 \mathrm{~K}, 5 \mathrm{~T}}$ and $\ln M_{5 \mathrm{~K}, 5 \mathrm{~T}}$ decrease linearly with increasing pressure with the rate of the latter listed in Table I.

The change of the magnetic properties upon changing the $\mathrm{Fe}$ atomic distances was already studied for the same $\mathrm{Fe}_{91} \mathrm{Zr}_{9}$ ribbon by hydrogen doping [64]. It was found that hydrogenation changed both $T_{C}$ and $T_{f}$ in the same sense as an increase of the $\mathrm{Zr}$ concentration, i.e., $T_{C}$ increases while $T_{f}$ decreases with increasing hydrogen content. It is the same effect that we have observed in the case of applying hydrostatic pressure (Fig. 6): The hydrogen atoms increase the Fe atomic volume, i.e., they act as negative pressure.

$\mathrm{Fe}_{88} \mathrm{Zr}_{12}$ behaves as a ferromagnet with only one magnetic transition temperature, i.e., the Curie temperature, $T_{C}$, as the pressure dependent $\mathrm{ZFC}$ and $\mathrm{FC}$ thermomagnetic curves show in Fig. 9. With increasing pressure, traces of a very weak spin freezing appear at low temperatures, but a quantitative evaluation is not possible. $T_{C}$ decreases linearly with increasing pressure with a rate of $-58.7 \mathrm{~K} \mathrm{GPa}^{-1}$ (listed in Table I). The low-field magnetization decreases with increasing pressure. Figure 10 shows the field dependence of magnetization measured at $5 \mathrm{~K}$ at different pressures denoted as labels. The magnetization decreases (but at an even lesser rate than for $\mathrm{Fe}_{91} \mathrm{Zr}_{9}$ ) with increasing pressure at any magnetic fields. Both $M_{5 \mathrm{~K}, 5 \mathrm{~T}}$ and $\ln M_{5 \mathrm{~K}, 5 \mathrm{~T}}$ decrease linearly with increasing pressure, with the rate of the latter listed in Table I.

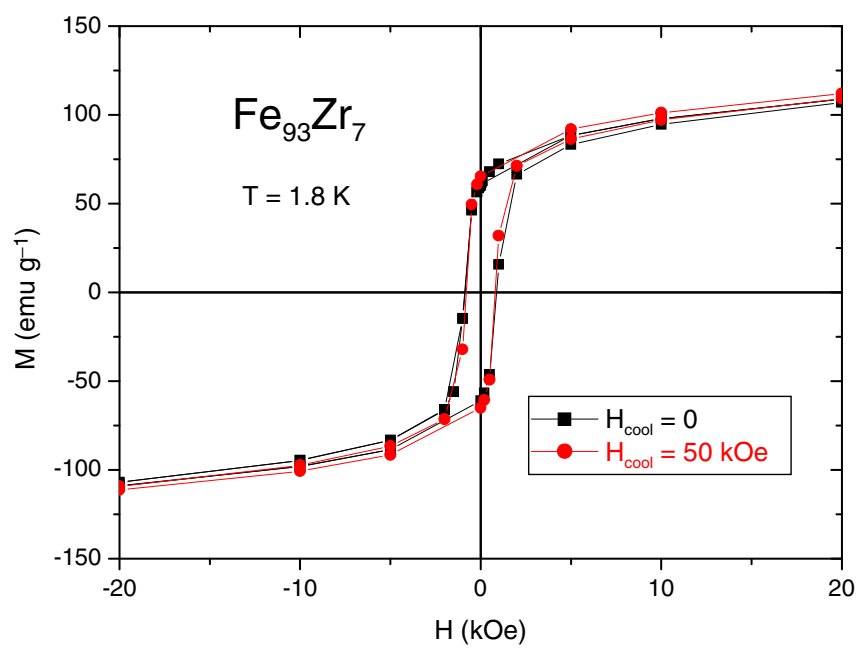

FIG. 5. Hysteresis loops measured between $\pm 50 \mathrm{kOe}$ for $\mathrm{Fe}_{93} \mathrm{Zr}_{7}$ after cooling in zero fields (black squares) and in $50 \mathrm{kOe}$ (red dots).

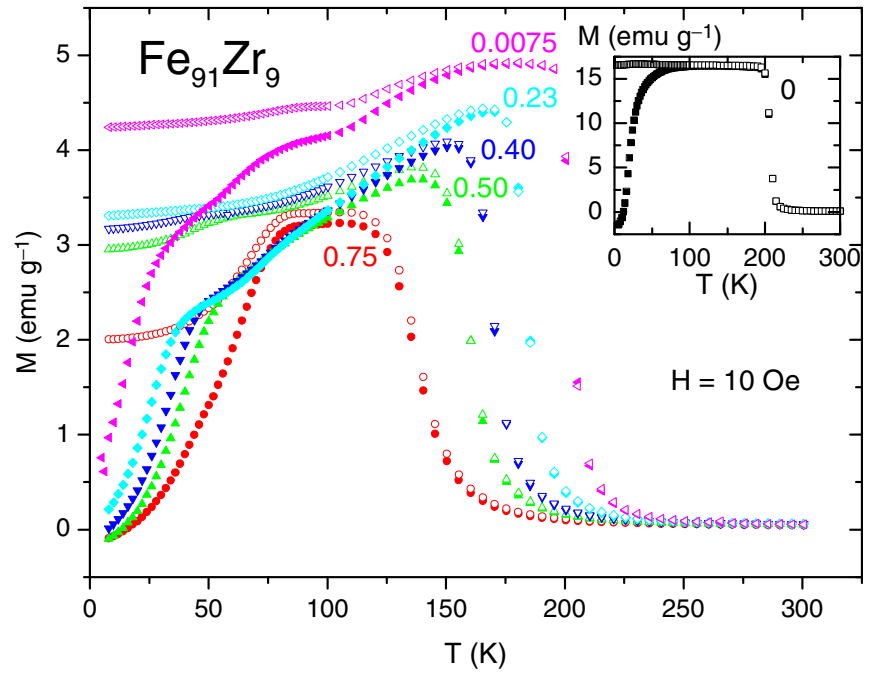

FIG. 6. Magnetization as a function of temperature for $\mathrm{Fe}_{91} \mathrm{Zr}_{9}$, measured after zero-field cooling (ZFC, solid symbols) and field cooling (FC, open symbols) in 10 Oe for different pressures. The pressure values are denoted as labels in GPa (0 means atmospheric pressure $=0.0001 \mathrm{GPa}$ ).

Finally, the pressure dependence of the saturation magnetization for $\mathrm{Co}_{91} \mathrm{Zr}_{9}$ was measured (figure is not included). This alloy has a high Curie temperature far above RT (see Table I); below RT, it shows no magnetic transition. It behaves as an ideal ferromagnet where the magnetization saturates even for relatively small fields (about $2 \mathrm{kOe}$ ). Though $M_{5 \mathrm{~K}, 5 \mathrm{~T}}$ and $\ln M_{5 \mathrm{~K}, 5 \mathrm{~T}}$ decrease linearly with increasing pressure, the rates are almost two orders of magnitude smaller than for $\mathrm{Fe}_{93} \mathrm{Zr}_{7}$ (the latter is listed in Table I). Though $T_{C}$ cannot be measured directly under pressure (the onset temperature of crystallization is lower than $T_{C}$; see Table I), an indirect measurement indicated that the pressure effect on $T_{C}$ approaches nearly zero in $\mathrm{Co}_{90} \mathrm{Zr}_{10}$ amorphous alloy [65].

The pressure dependence of the relative volume change $d V / V_{0}$ presented in Fig. 11 was fitted to the Bridgman [56]

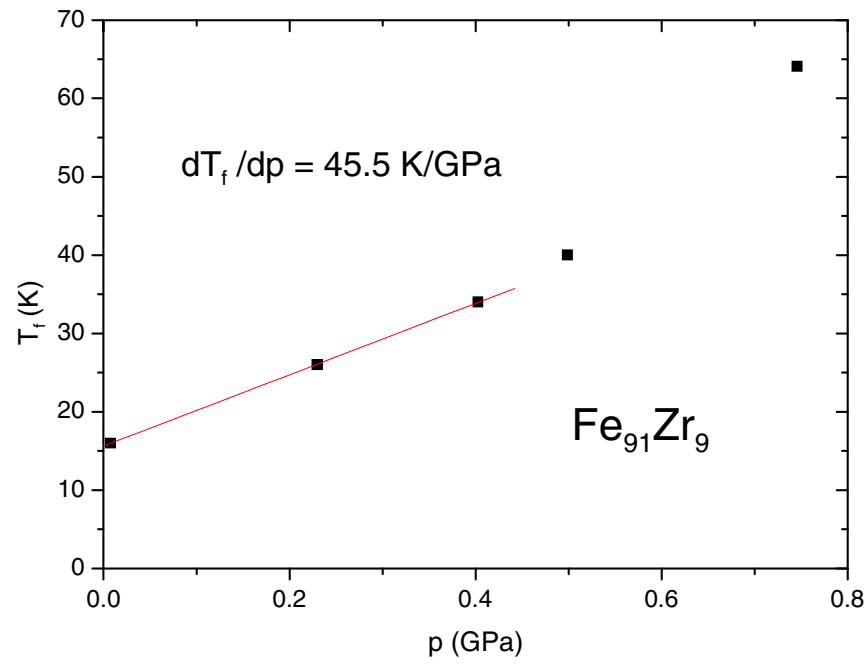

FIG. 7. Spin-freezing temperature, $T_{f}$, as a function of pressure for $\mathrm{Fe}_{91} \mathrm{Zr}_{9} . T_{f}$ increases with increasing pressure with a rate of $45.5 \mathrm{~K} \mathrm{GPa}^{-1}$ at low pressures. 


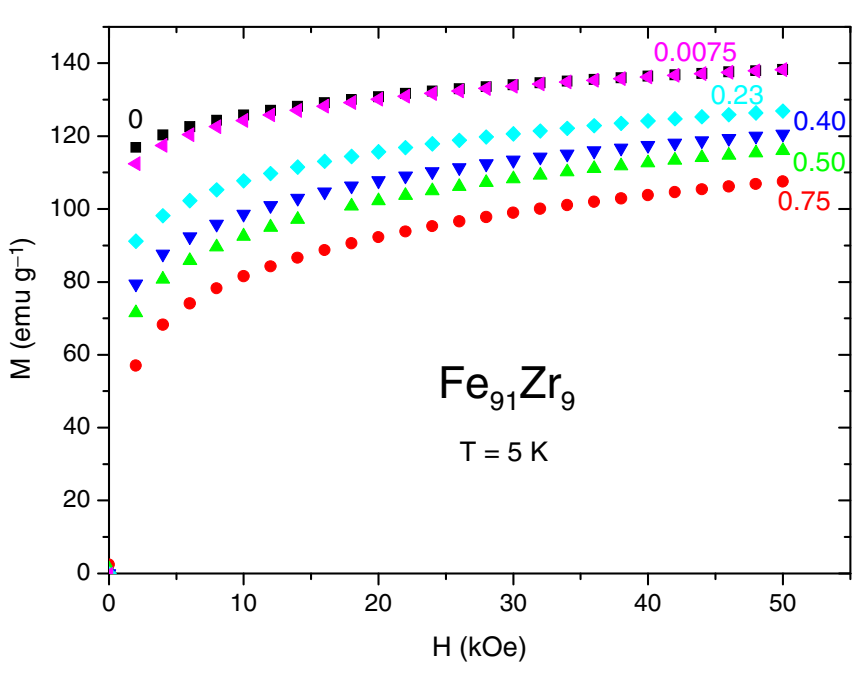

FIG. 8. Magnetization as a function of magnetic field for $\mathrm{Fe}_{91} \mathrm{Zr}_{9}$, measured at $5 \mathrm{~K}$ at different pressures denoted as labels in GPa (0 means atmospheric pressure $=0.0001 \mathrm{GPa}$ ).

equation of state, giving values of bulk moduli 151 and $152 \mathrm{GPa}$ for $\mathrm{Fe}_{91} \mathrm{Zr}_{9}$ and $\mathrm{Co}_{91} \mathrm{Zr}_{9}$, respectively (see Table I). The values of $\kappa$ and $a_{2}$ from the fit are also shown in Table I. The values of $B_{0}, \kappa$, and $a_{2}$ are very close to those found recently in Fe-Mn-B metallic glasses [66].

\section{MODEL}

\section{A. Without coupling}

In the cluster-glass model for the Fe-rich amorphous $\mathrm{Fe}-\mathrm{Zr}$ alloys [30,31], the magnetic clusters are embedded in an FM (infinite) matrix, and they are weakly coupled to it. At the critical $\mathrm{Zr}$ concentration $\left(\mathrm{Fe}_{93} \mathrm{Zr}_{7}\right)$, the coupling vanishes in the absence of the infinite FM matrix. In this model for

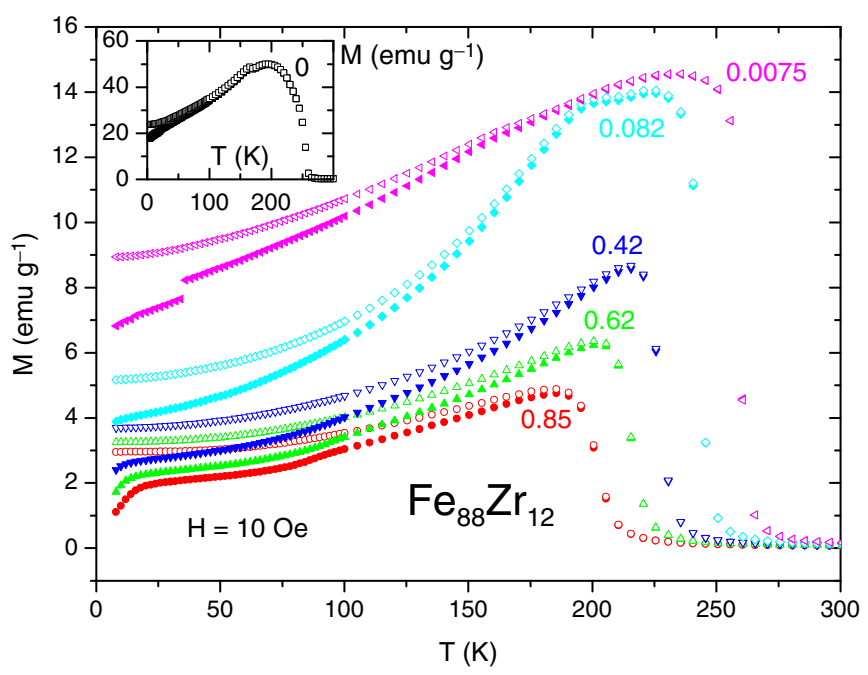

FIG. 9. Magnetization as a function of temperature for $\mathrm{Fe}_{88} \mathrm{Zr}_{12}$, measured after zero-field cooling (ZFC, solid symbols) and field cooling (FC, open symbols) in 10 Oe for different pressures. The pressure values are denoted as labels in GPa ( 0 means atmospheric pressure $=0.0001 \mathrm{GPa}$ ).

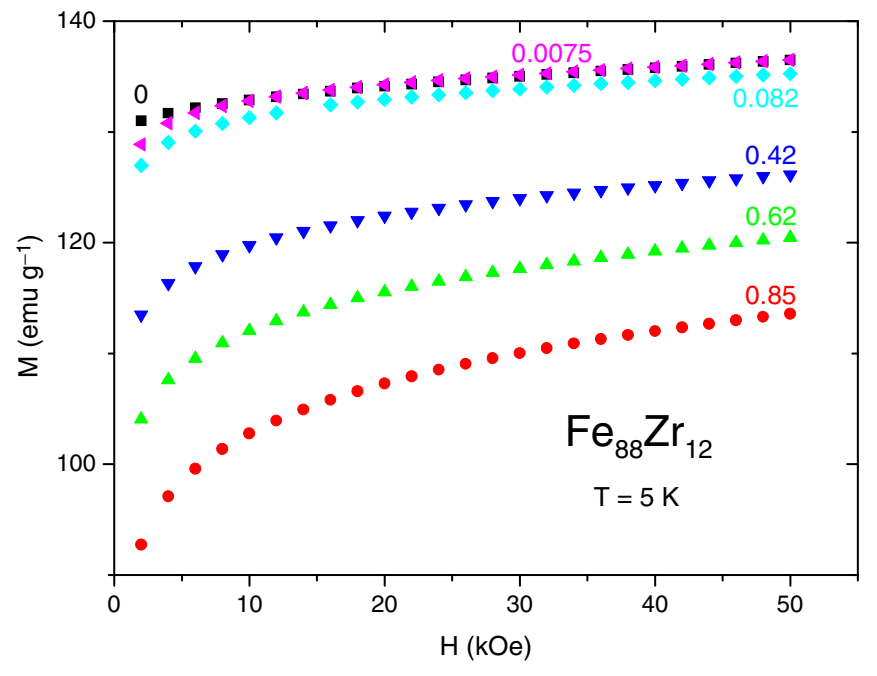

FIG. 10. Magnetization as a function of magnetic field for $\mathrm{Fe}_{88} \mathrm{Zr}_{12}$, measured at $5 \mathrm{~K}$ at different pressures denoted as labels in $\mathrm{GPa}(0$ means atmospheric pressure $=0.0001 \mathrm{GPa})$.

$\mathrm{Fe}_{93} \mathrm{Zr}_{7}$, the SG temperature, $T_{g}$, is determined entirely by the blocking temperature, $T_{B}$, of the clusters $\left(T_{B}\right.$ has, in fact, a distribution, but for simplicity now we examine one cluster or a monodisperse system). In fact, the interactions between the clusters cannot be neglected either in $\mathrm{Fe}_{93} \mathrm{Zr}_{7}$, as indicated by the frequency dependence of $T_{g}$, which can be well described by a Vogel-Fulcher-type expression with $T_{0}=100 \mathrm{~K}$ [67]. For simplicity, we do neglect them in this paper because we want to model the effect of the FM matrix on the spin-freezing properties. This effect will be taken into account by a mean-field model (see Sec. IV B). Since such a model is inadequate to describe the interactions between the clusters, we assume that the two types of interactions should be treated separately. In the usual treatment found in textbooks [68], $T_{B}$ is the temperature at which the thermal energy ( $k T_{B}$ where $k$ is Boltzmann's constant) overcomes

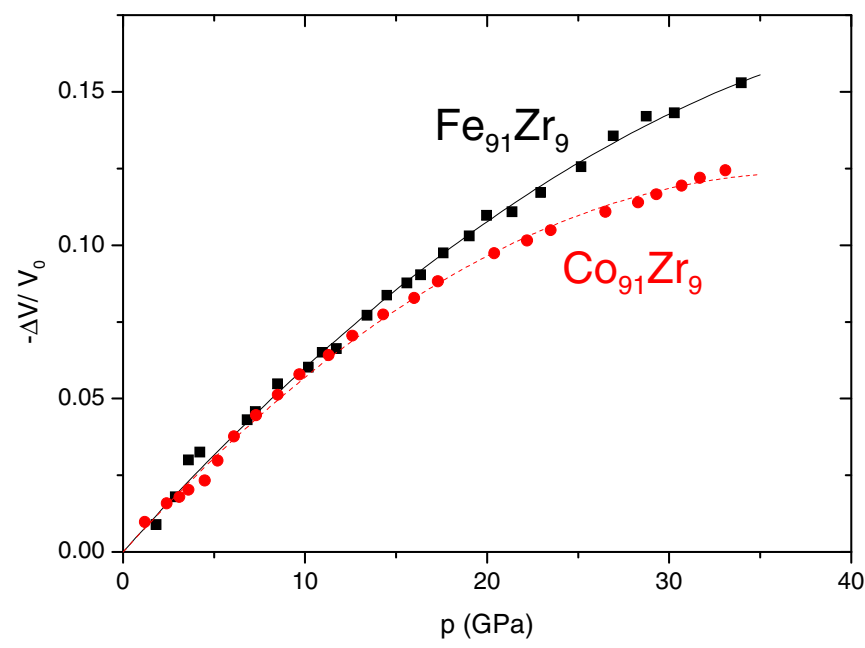

FIG. 11. Pressure dependence of relative volume change $\Delta V / V_{0}$ for $\mathrm{Fe}_{91} \mathrm{Zr}_{9}$ and $\mathrm{Co}_{91} \mathrm{Zr}_{9}$ metallic glasses at ambient temperature. The lines are fits to the Bridgman equation (see text). 
the energy barrier caused by the anisotropy of the cluster ( $K V$, where $K$ is the anisotropy constant and $V$ is the cluster volume). The origin of anisotropy can be crystal, shape, or stress anisotropy. As pointed out in the Introduction, it is difficult to interpret the meaning of anisotropy in our case. It is worth noting that the usual interpretation of blocking outlined above assumes a homogeneous rotation as the reversal mode of magnetization of the magnetic clusters. The magneticviscosity measurements made for the Fe-rich Fe-Zr metallic glasses [31], however, drew attention to the curling-type reversal mode of magnetization with the help of which the temperature and composition dependence of coercivity could be explained. The task is to find an expression for the energy barrier of the curling mode, which can replace $K V$ valid for homogeneous rotation.

In the curling mode, the spins are reversed at the expense of the exchange energy instead of the anisotropy energy, i.e., the neighboring spins will make an angle with respect to each other during reversal [69]. The ideal case is a cylinder (a special case of prolate spheroid), where all the spins are tangential to the lateral surface of the cylinder during reversal, thereby eliminating the stray-field energy. In this case, the reversal process is determined by the balance of the exchange energy and the energy of interaction with the external field (field energy). Based on this balance, the coercive field for the curling mode was calculated long ago for a prolate spheroid to be inversely proportional to the square of its size (characterized by its radius or diameter perpendicular to the rotation axis) [70,71]. The marked size dependence of coercivity for the curling mode is quite different from the size-independent coercivity obtained for homogeneous rotation.

The calculation of the energy barrier for curling is presented in the Appendix. First, we get for the coercive field,

$$
H_{c}=\frac{2 A}{R^{2} M_{s}}=\frac{2 M_{s}}{\left(R / R_{0}\right)^{2}},
$$

where $A$ is the exchange constant, $M_{s}$ is the saturation magnetization, $R$ is the radius of the particle (cylinder or sphere), and the notation $R_{0}=A^{1 / 2} / M_{s}$ was introduced in the second expression. It is the same formula for the size dependence of coercive field for curling as calculated before for a prolate spheroid [70,71]. Second, we determined the energy barrier for a particle of radius $R$ in the curling mode to be proportional to $\Delta E \propto A R$, which replaces the $\Delta E=K V$ term valid for homogeneous rotation.

\section{B. With coupling}

The coupling between the clusters and the infinite (FM) matrix is treated in the framework of a mean-field model. The physical picture behind this model is that the thermal energy $(k T)$ is aided by the mean-field of the FM matrix (representing its stray field) to facilitate the magnetization reversal of the clusters. The details of the calculations are presented in the Appendix. In the curling mode, we get for the energy barrier of a particle of radius $R$ without field,

$$
\Delta E=A R\left(1-\frac{R^{2} \lambda M_{s}^{2}}{2 A}\right)^{2}
$$

where $\lambda$ is the coupling constant. The blocking temperature, $T_{B}$, is determined by the condition $\Delta E=25 k T_{B}$,

$$
\begin{aligned}
T_{B} & =\frac{A R}{25 k}\left(1-\frac{R^{2} \lambda M_{s}^{2}}{2 A}\right)^{2}=\frac{A R}{25 k}\left(1-\frac{m_{s} \lambda M_{s}}{2 A R}\right)^{2} \\
& =\frac{\Delta E^{0}}{25 k}\left(1-\frac{E_{c}}{2 \Delta E^{0}}\right)^{2},
\end{aligned}
$$

where the cluster moment $m_{s}=R^{3} M_{s}$ was introduced in the second expression and the notations $\Delta E^{0}=A R$ and $E_{c}=$ $m_{s} \lambda M_{s}$ were applied in the third expression. Here $\Delta E^{0}$ is the energy barrier for curling without coupling, and $E_{c}$ is the coupling energy.

\section{DISCUSSION}

\section{A. Cluster behavior}

This simple model outlined above readily explains the decreasing trend of the SG temperature, $T_{g}$, for $\mathrm{Fe}_{93} \mathrm{Zr}_{7}$ with increasing pressure (Figs. 1 and 2). The hydrostatic pressure applied in the pressure cell shortens the separation between the $\mathrm{Fe}$ atoms, thereby increasing the number of Fe atoms carrying a reduced moment. It leads both to the decrease of the cluster size, $R$ (since some clusters decouple along the newly created lines of low-moment Fe atoms), and to the decrease of $A$ (since $J$ decreases on the Bethe-Slater curve). According to our assumption, $\mathrm{Fe}_{93} \mathrm{Zr}_{7}$ consists of only clusters without an FM matrix (with no interaction between the clusters); therefore, it is our model without coupling (Sec. IV A) that refers to it. Thus, the energy barrier (and hence $T_{B}$ ), which is proportional to $A R$ will decrease, decreasing $T_{g}$.

For $\mathrm{Fe}_{91} \mathrm{Zr}_{9}$, the spin-freezing temperature, $T_{f}$, changes with pressure in the opposite direction as $\mathrm{Fe}_{93} \mathrm{Zr}_{7}$, i.e., $T_{f}$ increases with increasing pressure. According to our model, above the critical $\mathrm{Zr}$ concentration $\left(x_{c}=7\right)$, the clusters become more and more coupled to the FM matrix; therefore, it is our model with coupling that refers to $\mathrm{Fe}_{91} \mathrm{Zr}_{9}$. Of course, the effect of pressure is the same for $\mathrm{Fe}_{91} \mathrm{Zr}_{9}$ as for $\mathrm{Fe}_{93} \mathrm{Zr}_{7}: R$ and $A$ decrease with increasing pressure. This would decrease $T_{f}$ if there were no coupling between the clusters and the matrix. However, the coupling characterized by $\lambda$ also decreases with increasing pressure, which leads to the increase of $T_{f}$ according to Eq. (3). The balance of these two opposite effects is a net increase of the spin-freezing temperature.

The absence of coupling between the clusters and the matrix makes a rough estimation of the exchange constant, $A$, possible. Measuring the magnetic viscosity [31], the magnetization change was recorded as a function of time at a given temperature. In this case, only clusters having $T_{B}$ equal to the measuring temperature contribute to the magnetization change since smaller clusters reverse before beginning the measurement and larger clusters remain blocked within the time scale of the measurement. Therefore, from these measurements the size dependence of the blocking temperature can be obtained. The result was that the cluster size varies linearly with $T_{B}$ for the amorphous $\mathrm{Fe}_{100-x} \mathrm{Zr}_{x}(7 \leqslant$ $x \leqslant 9)$ system [31]. Our current model predicts just the same dependence, so the magnetic-viscosity measurements experimentally support the validity of the model. According 
TABLE II. Parameters of the cluster-glass model for the $\mathrm{Fe}_{100-x} \mathrm{Zr}_{x}(x=7,8,9)$ metallic glasses. $T_{g}$ is the spin-glass temperature, $T_{C}$ is the Curie temperature, $A$ is the exchange constant, $\Delta E^{0}$ is the energy barrier for thermal blocking, and $E_{c}$ is the coupling energy between the clusters and the FM matrix, $N_{\mathrm{cl}}$ is the number of atoms in a cluster, $\mu$ is the magnetic moment per Fe atom, and $H_{m}$ is the effective coupling field (for explanation see text).

\begin{tabular}{lccc}
\hline \hline & $\mathrm{Fe}_{93} \mathrm{Zr}_{7}$ & $\mathrm{Fe}_{92} \mathrm{Zr}_{8}$ & $\mathrm{Fe}_{92} \mathrm{Zr}_{9}$ \\
\hline$T_{g}, T_{C}(\mathrm{~K})$ & 110 & $174[30]$ & 205 \\
$A\left(10^{-7} \mathrm{erg} \mathrm{cm}{ }^{-1}\right)$ & 2.3 & 3.6 & 4.3 \\
$\Delta E^{0}\left(10^{-13} \mathrm{erg}\right)$ & 0.81 & 1.62 & 4.3 \\
$E_{c}\left(10^{-13} \mathrm{erg}\right)$ & 0 & 0.94 & 2.57 \\
$N_{\mathrm{cl}}[30]$ & 20000 & 30000 & 70000 \\
$\mu\left(\mu_{B} / \mathrm{Fe}\right)$ & 1.4 & 1.5 & 1.6 \\
$H_{m}(\mathrm{Oe})$ & 0 & 225 & 247 \\
\hline \hline
\end{tabular}

to these measurements, for $\mathrm{Fe}_{93} \mathrm{Zr}_{7}$ a cluster with a radius of $R=3.5 \mathrm{~nm}$ has a blocking temperature of $T_{B}=24 \mathrm{~K}$. The exchange constant for $\mathrm{Fe}_{93} \mathrm{Zr}_{7}$ can be estimated to be $A=25 k T_{B} / R=2.310^{-7} \mathrm{erg} \mathrm{cm}^{-1}$. This method to estimate $A$ for $\mathrm{Fe}_{92} \mathrm{Zr}_{8}, \mathrm{Fe}_{91} \mathrm{Zr}_{9}$, and $\mathrm{Fe}_{88} \mathrm{Zr}_{12}$ cannot be used because the coupling constant, $\lambda$, is not known. However, $A$ is known to be proportional to the exchange integral, $J$, and hence to the Curie temperature, $T_{C}$ [72], which makes an estimation of $A$ for $\mathrm{Fe}_{92} \mathrm{Zr}_{8}, \mathrm{Fe}_{91} \mathrm{Zr}_{9}$, and $\mathrm{Fe}_{88} \mathrm{Zr}_{12}$ possible by scaling (see Table II). Once $A$ is known, the energy barrier, $\Delta E^{0}$, and the coupling energy, $E_{c}$, can be estimated for $\mathrm{Fe}_{92} \mathrm{Zr}_{8}, \mathrm{Fe}_{91} \mathrm{Zr}_{9}$, and $\mathrm{Fe}_{88} \mathrm{Zr}_{12}$ by the expression

$$
E_{c}=2 \Delta E^{0}\left(1-\sqrt{\frac{25 k T_{B}}{\Delta E^{0}}}\right),
$$

which is obtained by rearranging Eq. (3). The data for the alloys $\left(R, T_{B}\right)$ are taken from viscosity measurements [31], and the values of $E_{c}$ are listed in Table II. The coupling energy, $E_{c}$, can be written as $E_{c}=m_{s} \lambda M_{s}=m_{s} H_{m}$, where $H_{m}=\lambda M_{s}$ is the effective coupling field. Estimating the values for the cluster moment and saturation magnetization from magnetization measurements, the effective coupling field is calculated to be of the order of a few hundred Oe (see Table II).

We obtained plausible values for the exchange constant of our studied alloys: $A \approx 2-410^{-7} \mathrm{erg} \mathrm{cm}^{-1}$. For $\alpha-\mathrm{Fe}, A$ is estimated to be of the order of $10^{-6} \mathrm{erg} \mathrm{cm}^{-1}$ [73]; therefore, our alloys having about a tenth of the Curie point of $\alpha$-Fe are expected to have an $A$ of one order of magnitude smaller. Only limited data are found in the literature as to the spin-wave stiffness constant, $D_{\text {sc }}$, for Fe-Zr metallic glasses ( $A$ is usually calculated from $D_{\mathrm{sc}}$ ). For $\mathrm{Fe}_{91} \mathrm{Zr}_{9}$ [74] and $\mathrm{Fe}_{90} \mathrm{Zr}_{10}[74,75]$, the spin-wave stiffness constant takes the values of $D_{\text {sc }}=29$ and $31 \mathrm{meV}^{2}$, respectively. A value of $D_{\mathrm{sc}}=37 \mathrm{meV} \AA^{2}$ is given elsewhere for $\mathrm{Fe}_{90} \mathrm{Zr}_{10}$ [76]. The values of $D_{\mathrm{sc}}$ for $\alpha-\mathrm{Fe}$ scatter around 280 to $311 \mathrm{meV}^{2}$ [77], reflecting the tenfold difference in the Curie points.

To understand the complex magnetic behavior observed in the Fe-rich amorphous $\mathrm{Fe}-\mathrm{Zr}$ alloys at low temperatures, it is necessary to explain the temperature and concentration dependence of susceptibility (magnetization at constant fields) and coercivity within the framework of the same model. The bifurcation of the ZFC and FC magnetization curves with decreasing temperature due to spin freezing (Figs. 1, 6, and 9) can easily be interpreted by only assuming a blocking-temperature distribution, $f\left(T_{B}\right)$ [30]. The type of magnetization reversal is indifferent in this case: The plausible assumption of a volume distribution for the clusters is enough for the presence of $f$ $\left(T_{B}\right)$. The only difference is that $T_{B}$ is proportional to the radius (or diameter) of the clusters for curling, $T_{B} \propto A R$ (as we showed in Sec. IV), while it is proportional to the cluster volume, $T_{B} \propto K V$, for homogeneous rotation.

The second point to be explained is the composition dependence of the spin-freezing temperature, $T_{f}$, i.e., why $T_{f}$ decreases with increasing $\mathrm{Zr}$ content, $x$. Here, the dominant factor is the coupling between the clusters and the FM matrix: $\lambda$ increases with $x$ going in the direction of the FM behavior, and according to Eq. (3), it leads to the decrease of $T_{B}$. For curling, this decrease should compensate the increase of $T_{B}$ originating from the increase of the cluster size, $R$, with $x$ deduced from magnetic viscosity measurements [31]. For homogeneous rotation, the same explanation holds with the exception that here $T_{B}$ increases with the cluster volume, $V$, when increasing $x$. Consequently, the type of reversal cannot be decided from the composition dependence of $T_{f}$.

The third point to be interpreted is the composition dependence of coercivity, i.e., the increasing magnetic hardness with decreasing $\mathrm{Zr}$ content. Our model offers a simple explanation for this dependence: As deduced from magnetic-viscosity measurements [31], the cluster size, $R$, decreases with decreasing $x$, resulting in the increase of $H_{c}$ according to Eq. (1). Since both $A$ and $M_{s}$ in Eq. (1) decrease with decreasing $x$, their ratio can be regarded as composition independent. In contrast, $H_{c}$ is independent of the cluster size for homogeneous rotation and depends only on the anisotropy constant, $K$, and the saturation magnetization, $M_{s}$, of the clusters $\left(H_{c}=2 K / M_{s}\right)$. Assuming this reversal mode, the increasing magnetic hardness with decreasing $x$ can be explained by the increase of $K$ and/or the decrease of $M_{s}$ with decreasing $x$. The latter is an experimental fact; however, this dependence is not so strong to account for the almost exponential increase of $H_{c}$ with decreasing $x$. The dependence of $K$ on $x$ is not known; even the origin of an anisotropy of a cluster in an amorphous system is questionable (at most, shape anisotropy can be imagined but in that case it is difficult to interpret its composition dependence). This argument, rather, supports the curling mode, but it is not decisive.

The fourth point is the explanation of the temperature dependence of coercivity. As a starting point, let us first examine the monodisperse model system where only one blocking temperature exists. The temperature dependence of $H_{c}$ is determined by the balance of the energy barrier for curling [Eq. (2)] and the thermal energy:

$$
A R\left(1-\frac{R^{2} H_{c} M_{s}}{2 A}\right)^{2}=25 k T .
$$

Expressing $H_{c}$, we get for curling,

$$
H_{c}=\frac{2 A}{R^{2} M_{s}}\left[1-\left(\frac{25 k T}{A R}\right)^{1 / 2}\right],
$$

where the prefactor $H_{c}^{0}=2 A /\left(R^{2} M_{S}\right)$ is the coercive field at $T \ll T_{B}$, which we already obtained in Eq. (1). The analogous 
expression for homogeneous rotation is [78],

$$
H_{c}=\frac{2 K}{M_{s}}\left[1-\left(\frac{25 k T}{K V}\right)^{1 / 2}\right] .
$$

Though this temperature dependence of $H_{c}$ is seemingly similar to the observed one, it is of questionable reality since the presence of a $T_{B}$ distribution significantly modifies it. At a given temperature, $T$, only clusters with $T_{B}>T$ contribute to the coercivity since they are blocked. These clusters can very roughly be characterized by an average blocking temperature, $\bar{T}_{B}=A \bar{R}(>T)$ for curling or $\bar{T}_{B}=K \bar{V}$ for homogeneous rotation, thereby determining $H_{c}$. With decreasing temperature, smaller and smaller clusters with smaller and smaller $T_{B}$ contribute to $H_{c}$, resulting in $T \propto \bar{T}_{B}$. Therefore, the explicit temperature dependence of $H_{c}$, shown inside the brackets in Eqs. (3) and (4), is almost eliminated, and $H_{c}$ will be determined dominantly by the prefactors. With decreasing temperature, the decreasing $\bar{R}$ causes the steep increase of $H_{c}$ due the inverse quadratic dependence of $H_{c}$ on $R$ in the curling mode [see $H_{c}^{0}$ in Eq. (4)]. $M_{S}$ slightly increases with decreasing temperature in the actual narrow temperature range ( 5 to $60 \mathrm{~K}$ ), probably together with $A$, thereby their ratio can be regarded as temperature independent. For homogeneous rotation, the only explanation for the steep increase of $H_{c}$ with decreasing temperature would be the assumption that the cluster anisotropy, $K$, abruptly increases with decreasing temperature [Eq. (5)]; however, it is difficult to justify such an assumption. Therefore, the dependence of $H_{c}$ on $T$ is a decisive support for the curling reversal mode.

It is remarkable that for $\mathrm{Fe}_{93} \mathrm{Zr}_{7}$, the coercivity depends only weakly on the applied pressure, though $H_{c}$ shows the same abrupt increase with decreasing temperature at different constant pressures as it behaves at 1 bar (Fig. 4). Applying a pressure in the range of 0 to $1 \mathrm{GPa}$, the cluster size, $R$, should decrease, but this effect should be much less than that caused by decreasing the $\mathrm{Zr}$ content. According to Eq. (1), $H_{c}$ should increase moderately with pressure, as observed for $\mathrm{Fe}_{93} \mathrm{Zr}_{7}$ at $10 \mathrm{~K}$ (main figure in Fig. 4). Since both $A$ and $M_{s}$ in Eq. (1) are also expected to decrease with pressure, their ratio remains only weakly pressure dependent, making unpredictable the exact pressure dependence of $H_{c}$. Indeed, as seen in the inset of Fig. 4, $H_{c}$ can even decrease with pressure at higher temperatures, but in any case its pressure dependence remains weak.

\section{B. Behavior of the FM matrix}

Shirakawa et al. [35] analyzed the pressure dependence of $T_{C}$ for the Fe-rich Fe-Zr metallic glasses using the theory of Wagner and Wohlfarth, who discussed the magnetism of inhomogeneous FM alloys on the basis of the LandauGinzburg model $[79,80]$. This theory is developed within the weak itinerant-electron model. According to the theory, the pressure derivative of $T_{C}$ for inhomogeneous FM alloys is given by

$$
d T_{C} / d p=-\chi_{1} T_{C}+\chi_{2} T_{C}^{2},
$$

where $\chi_{1}(>0)$ and $\chi_{2}(>0)$ are parameters. Shirakawa et al. showed that the values of $d T_{C} / d p$ and $T_{C}$ measured for

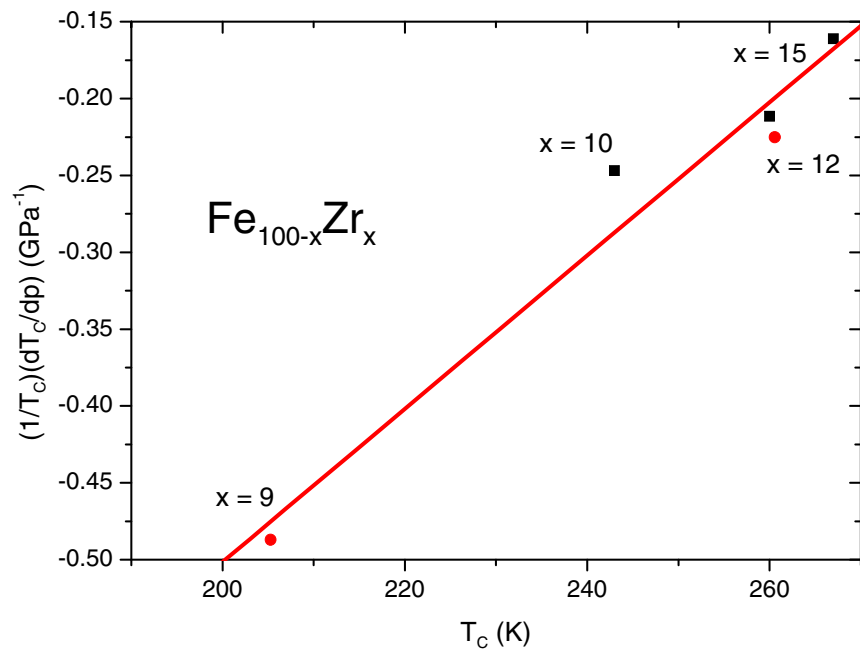

FIG. 12. $\left(1 / T_{C}\right)\left(d T_{C} / d p\right)$ as a function of $T_{C}$ for $\mathrm{Fe}_{100-x} \mathrm{Zr}_{x}$. The labels show the $\mathrm{Zr}$ concentrations. Squares are data of Shirakawa et al. (see Ref. [35]); dots are taken from present work. The line is a linear fit to all points.

$\mathrm{Fe}_{100-x} \mathrm{Zr}_{x}(x=7,10,12$, and 15) excellently satisfy Eq. (6). They also showed that these data do not fit equally well the relationship $d T_{C} / d p=-\alpha / T_{C}(\alpha$ is a positive parameter and is assumed to be constant within one alloy system) proposed by Wohlfarth [81] for homogeneous FM alloys (Fe-Ni crystalline Invar alloys) in terms of the weak itinerant model. The results of our investigation confirm the conclusion of Shirakawa et al. that the amorphous $\mathrm{Fe}-\mathrm{Zr}$ alloys are magnetically inhomogeneous. Figure 12 shows $\left(1 / T_{C}\right) d T_{C} / d p$ as a function of $T_{C}$ for our $\mathrm{Fe}_{91} \mathrm{Zr}_{9}$ and $\mathrm{Fe}_{88} \mathrm{Zr}_{12}$ alloys, together with the results of Shirakawa et al. for $\mathrm{Fe}_{100-x} \mathrm{Zr}_{x}(x=7,10,12$, and 15). The agreement is excellent for $\mathrm{Fe}_{88} \mathrm{Zr}_{12}$, which is the only alloy with the same nominal composition measured by the two groups. The point of Shirakawa et al. for $\mathrm{Fe}_{93} \mathrm{Zr}_{7}$ is excluded from the present analysis since this alloy is not FM, as discussed before. As seen in Fig. 12, the data are in line with Eq. (6), supporting the inhomogeneous nature of these amorphous alloys $\left(\chi_{1}=1.50 \mathrm{GPa}^{-1}\right.$ and $\left.\chi_{2}=0.0050 \mathrm{~K} / \mathrm{GPa}\right)$. However, it is to be noted that a linear relationship between $d T_{C} / d p$ and $1 / T_{C}$ (which was predicted for magnetically homogeneous alloys) might also not be excluded. The reason of this uncertainty is probably the very narrow concentration range available in our case $(x=7$ to $15 \%)$. Since there are a lot of other indications pointing to an inhomogeneous magnetic structure in these alloys, we can only conclude that the pressure dependence of $T_{C}$ as a function of composition does not contradict to the inhomogeneous model.

The theory predicts a relationship between the pressure derivatives of saturation magnetization, $M_{s}$, and Curie temperature, $T_{C}$,

$$
\frac{d \ln M_{s}}{d p}=\frac{d \ln T_{C}}{d p}-\frac{5}{6} \kappa-\frac{d \ln B}{d p},
$$

where $B$ is a second Landau coefficient in the expansion of the thermodynamic potential and the last term is considered to be small. While no data on pressure derivatives of $M_{s}$ can be found in the literature for this alloy system, the 
validity of Eq. (7) can now be checked with our results. As evident from Table I, the compressibility measured for $\mathrm{Fe}_{91} \mathrm{Zr}_{9}$ is small $\left(\kappa=6.6410^{-3} \mathrm{GPa}^{-1}\right)$; therefore, it is the first term $\left(d \ln T_{C} / d p=-0.60 \mathrm{GPa}^{-1}\right)$ that dominates on the right side of Eq. (7) compared to the second term $[-(5 / 6) \kappa=$ $\left.-0.0053 \mathrm{GPa}^{-1}\right]$. The same is true for $\mathrm{Fe}_{88} \mathrm{Zr}_{12}$ if we use the compressibility value obtained for $\mathrm{Fe}_{91} \mathrm{Zr}_{9}$, which should be a good approximation (see also Table I). Taking the values of $d \ln M_{s} / d p$ (Table I) measured for $\mathrm{Fe}_{88} \mathrm{Zr}_{12}$ and $\mathrm{Fe}_{91} \mathrm{Zr}_{9}$ $\left(d \ln M_{5 \mathrm{~K}, 5 \mathrm{~T}} / d p=-0.23\right.$ and $-0.34 \mathrm{GPa}^{-1}$, respectively), Eq. (7) is valid for $\mathrm{Fe}_{88} \mathrm{Zr}_{12}\left(-0.23 \mathrm{GPa}^{-1} \approx-0.26 \mathrm{GPa}^{-1}\right.$ $\left.-0.0053 \mathrm{GPa}^{-1}\right)$, while the discrepancy is greater for $\mathrm{Fe}_{91} \mathrm{Zr}_{9}$ $\left(-0.34 \mathrm{GPa}^{-1}\right.$ compared to $\left.-0.60 \mathrm{GPa}^{-1}-0.0053 \mathrm{GPa}^{-1}\right)$. This discrepancy might be associated with the increasing deviation of the matrix from an ideal FM one upon decreasing $\mathrm{Zr}$ content due to cluster formation. In a very recent paper [82], electronic structure and magnetic properties for $\mathrm{ZrFe}_{2}$ with a cubic Laves phase were calculated based on the density functional theory. According to the calculations, the magnetic moment $(m)$ decreases under pressure in the vicinity of the experimental lattice constant with $d \ln m / d p=-0.038 \mathrm{GPa}^{-1}$. This value is about the tenth of those obtained for our Fe-rich amorphous $\mathrm{Fe}-\mathrm{Zr}$ alloys. The difference might be related to the much greater $\mathrm{Zr}$ content of the crystalline $\mathrm{ZrFe}_{2}$ phase. The amorphous or crystalline nature of the material may not be the dominant factor to determine the Fe magnetic moment in Fe-rich environments since it is mainly influenced by the atomic volume.

Finally, we discuss how the critical Fe atomic volume postulated on the basis of ambient-pressure density measurements performed for Fe-rich Fe-TM metallic glasses [27,28] is reflected in our pressure-dependent magnetic measurements made for amorphous $\mathrm{Fe}_{100-x} \mathrm{Zr}_{x}(x=7,9,12)$ alloys. For comparison, the pressure dependence of saturation magnetization for the $\mathrm{Co}_{91} \mathrm{Zr}_{9}$ metallic glass was also measured since in this case no drastic change in the exchange integral, $J$, is expected upon decreasing the interatomic distance by pressure. Indeed, the logarithmic decrease of $M_{s}$ with pressure is about 50 times smaller for $\mathrm{Co}_{91} \mathrm{Zr}_{9}$ than for the $\mathrm{Fe}-\mathrm{Zr}$ glasses studied in this work (Table I). The pressure coefficient of $M_{s}$ for the $\mathrm{Fe}-\mathrm{Zr}$ glasses is even several times larger than that of the $\mathrm{Fe}_{60} \mathrm{Mn}_{20} \mathrm{~B}_{20}$ and $\mathrm{Fe}_{56} \mathrm{Mn}_{24} \mathrm{~B}_{20}$ glasses $\left(d \ln M_{5 \mathrm{~K}, 5 \mathrm{~T}} / d p=\right.$ -0.15 and $-0.13 \mathrm{GPa}^{-1}$, respectively) [66]. The same is true (Table I) for the pressure coefficient of the Curie temperature, $T_{C}$, which is $d T_{C} / d p=-31$ and $-32 \mathrm{~K} \mathrm{GPa}^{-1}$ for $\mathrm{Fe}_{60} \mathrm{Mn}_{20} \mathrm{~B}_{20}$ and $\mathrm{Fe}_{56} \mathrm{Mn}_{24} \mathrm{~B}_{20}$, respectively. The best measure of the sensitivity of magnetization (magnetic moment) on volume changes is the Grüneisen parameter $(\Gamma=$ $\left.d \ln M_{s} / d \ln V\right) . \Gamma$ is orders of magnitude larger for the Fe-rich amorphous $\mathrm{Fe}-\mathrm{Zr}$ glasses than for $\mathrm{Co}_{91} \mathrm{Zr}_{9}(\Gamma=74,51$, and 34 for $\mathrm{Fe}_{93} \mathrm{Zr}_{7}, \mathrm{Fe}_{91} \mathrm{Zr}_{9}$, and $\mathrm{Fe}_{88} \mathrm{Zr}_{12}$, respectively, compared to $\Gamma=1.18$ for $\mathrm{Co}_{91} \mathrm{Zr}_{9}$; see Table I). These $\Gamma$ values obtained for the $\mathrm{Fe}-\mathrm{Zr}$ glasses are even much larger than those found for the Fe-Mn-B glasses $(\Gamma \approx 20)$. The extreme sensitivity of the magnetic properties to pressure for the Fe-rich $\mathrm{Fe}-\mathrm{Zr}$ glasses experimentally supports the widely accepted picture (see references given in the Introduction), according to which the average $\mathrm{Fe}$ atomic volume in these amorphous alloys is fairly close to the critical volume, $V_{f c c *} \approx 11.7 \AA^{3}$, separating the so-called LS and HS state of fcc Fe [8].

\section{CONCLUSIONS}

A cluster-glass model based on the curling-type reversal mode of magnetization is proposed for the comprehensive explanation of the low-temperature magnetic anomalies observed in general for Fe-rich amorphous $\mathrm{Fe}-T M$ alloys and specifically for Fe-rich $\mathrm{Fe}_{100-x} \mathrm{Zr}_{x}$ ( $x=7$ to 12 ) metallic glasses studied in this work. The only assumption is that the clusters should have a size distribution and that they are weakly coupled to the FM matrix. This picture explains not only the temperature and composition dependence of the coercivity, as suggested earlier [31], but also those of the susceptibility. Moreover, it is able to interpret the opposite pressure dependence of the SG temperature, $T_{g}$ (for $\mathrm{Fe}_{93} \mathrm{Zr}_{7}$ ), and the spin-freezing temperature, $T_{f}$ (for $\mathrm{Fe}_{91} \mathrm{Zr}_{9}$ ), which were systematically measured in the present study. The pressure dependence of the Curie temperature that is one of the characteristics of the FM matrix is in line with literature results and points to the inhomogeneous magnetic nature of the matrix. The pressure dependence of the saturation magnetization, $M_{s}$, also characteristic to the FM matrix, satisfies well the predictions of the itinerant-electron model.

The main difference between this treatment and those using the traditional cluster picture is that here the energy barrier for thermal blocking of the clusters is not determined by any kind of anisotropy $(K V)$ rather by the resistance of neighboring spins against misalignment due to the exchange interaction between them. In this case, the energy barrier for curling is calculated to be proportional to $A R$. This result is independent from our concrete model for the Fe-rich $\mathrm{Fe}-\mathrm{Zr}$ glasses: It generally gives the value of the energy barrier for thermal blocking of a particle of radius $R$ reversing its magnetization by curling. As far as we know, this expression has not been published so far; however, the particle-size dependence of coercivity (which can also be deduced from our result) was calculated before in the frame work of a more general treatment $[70,71]$.

A delicate point of the model is the origin of the clusters. There is a lot of experimental and theoretical evidence (see Introduction) that the average $\mathrm{Fe}-\mathrm{Fe}$ distance in these amorphous alloys is fairly close to a critical distance separating the LS (for higher density) and HS (for lower density) state of fcc Fe. Our pressure-dependent magnetic measurements, together with a compressibility study on $\mathrm{Fe}_{91} \mathrm{Zr}_{9}$ and $\mathrm{Co}_{91} \mathrm{Zr}_{9}$, revealed the extreme sensitivity of the magnetic properties with volume change for $\mathrm{Fe}_{91} \mathrm{Zr}_{9}$, in contrast to the almost pressure-independent magnetic behavior of $\mathrm{Co}_{91} \mathrm{Zr}_{9}$. Due to the amorphous nature of these alloys, there is a wide distribution of $\mathrm{Fe}-\mathrm{Fe}$ atomic distances around the average value. The clusters are imagined to be separated from the FM matrix by $\mathrm{Fe}$ atoms with reduced magnetic moments that form a percolating network. The less the $\mathrm{Zr}$ content, the more are these Fe atoms with reduced moments, decreasing the cluster size. At the critical concentration $\left(\mathrm{Fe}_{93} \mathrm{Zr}_{7}\right)$, the material consists of only clusters without an FM matrix.

\section{ACKNOWLEDGMENTS}

This work was supported by the Országos Tudományos Kutatási Alapprogramok (Hungarian Scientific Research Fund) under Grant No. OTKA K 101456. The fruitful discussion with Dr I. Bakonyi and his providing us with the amorphous 
$\mathrm{Fe}_{91} \mathrm{Zr}_{9}$ and $\mathrm{Co}_{91} \mathrm{Zr}_{9}$ samples are gratefully acknowledged. We thank L. Bujdosó for the preparation of the other samples. The use of the Extreme Condition Science Infrastructure (ECSI) at PETRA III is acknowledged for gas loading samples and ruby fluorescence measurements.

\section{APPENDIX}

\section{Without coupling}

To obtain the energy barrier for curling, we consider the exchange energy per unit volume for an infinite cylinder using a $(r, \phi, z)$ cylindrical coordinate system, where the magnetization changes occur by spin rotation from the $z$ axis (direction of the external field) in a plane perpendicular to the radius [70],

$$
E_{e x}=A\left[(d \omega / d r)^{2}+\left(1 / r^{2}\right) \sin ^{2} \omega\right],
$$

where $A$ is the exchange constant and $\omega$ is the angle between the spin direction and the $z$ axis. Let us take a look at the second term of the exchange energy since it can also be derived descriptively, and this will shed light on the underlying physics. Let us consider the case when all spins are perpendicular to the $z$ axis (rotation axis) of a cylinder of radius $R\left(\omega=90^{\circ}\right)$ and tangential to its lateral surface, as shown in Fig. 13(a). The exchange energy of two neighboring spins with an angle $\phi$ between them is given by $E_{e x}=J S^{2} \phi^{2}$, where $J$ is the exchange integral and $S$ is the spin quantum number. $\phi$ can be written as $\phi=2 \pi / N$ since the total change in angle of the spins in going around the ring is $2 \pi$ and the number of atoms in such a ring is $N=2 \pi R / a$, where $a$ is the distance between nearest neighbors. Thus $\phi=a / R$, giving for the exchange energy of neighboring spins $E_{e x}=J S^{2} a^{2} / R^{2}$. For other $\omega$ values, taking intuitively the projection of spins onto a plane perpendicular to the $z$ axis, we get $E_{e x}=\left(J S^{2} a^{2} / R^{2}\right) \sin ^{2} \omega$. Dividing by $a^{3}$, the exchange energy per unit volume is given by

$$
E_{e x}=\frac{J S^{2}}{a} \frac{\sin ^{2} \omega}{R^{2}} \propto A \frac{\sin ^{2} \omega}{R^{2}},
$$

where $A=n J S^{2} / a$ by definition, with $n$ denoting the number of atoms in the unit cell [83] ( $n=2$ and 4 for body-centeredcubic (bcc) and fcc crystal structures, respectively). This expression is the same as the second term in Eq. (A1).
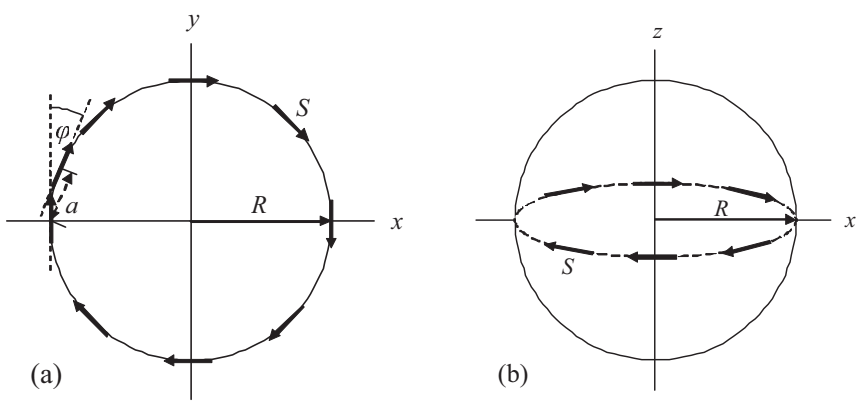

FIG. 13. Schematic view of spins $S$ during curling: (a) cross section normal to the $z$ axis after $\omega=90^{\circ}$ rotation from the $+z$ direction ( $\varphi$ is the angle between neighboring spins and $a$ is their distance); (b) a sphere of radius $R$ reversing magnetization by curling where the spins at the surface in the equatorial plane are shown.
To calculate the energy barrier for curling, we have to minimize the total energy per unit volume $\left(E_{\text {tot }}\right)$ consisting of exchange energy $\left(E_{e x}\right)$ and field energy $\left(E_{H}\right)$ terms:

$$
E_{\mathrm{tot}}=E_{e x}+E_{H}=A \frac{\sin ^{2} \omega}{R^{2}}+H M_{s} \cos \omega,
$$

where $H$ is the external field and $M_{S}$ is the saturation magnetization. The energy difference per unit volume $(\Delta E)$ between the two minima (i.e., the energy barrier per unit volume) is given by

$$
\Delta E=\frac{A}{R^{2}}\left(1-\frac{R^{2} H M_{s}}{2 A}\right)^{2}=\frac{A}{R^{2}}\left(1-\frac{H}{\frac{2 A}{R^{2} M_{s}}}\right)^{2} .
$$

From Eq. (A3), we get for the coercive field,

$$
H_{c}=\frac{2 A}{R^{2} M_{s}}=\frac{2 M_{s}}{\left(R / R_{0}\right)^{2}},
$$

where the notation $R_{0}=A^{1 / 2} / M_{s}$ was introduced in the second expression. Thus, we obtained the same formula for the size dependence of coercive field for curling as calculated before for a prolate spheroid [70,71].

Our calculation leads to another important result not published before, viz. it gives the energy barrier per unit volume without field for the curling mode: $\Delta E=A / R^{2}$. The quantity we are really interested in is the energy barrier for a particle of volume $V$, which determines the blocking temperature, $T_{B}$, for curling.

Multiplying with the volume, which is, as a first approximation, proportional to $R^{3}$, we get for a particle of volume $V$ in the curling mode: $\Delta E \propto A R$. To obtain a more precise result, the exchange energy per unit volume has to be integrated for the volume of the prolate spheroid. This calculation was already done [84] in order to obtain the critical size for a single-domain particle. Applying this result for our case, the energy barrier of a sphere of radius $R$ will be [Fig. 13(b)],

$$
\Delta E=\frac{8 \pi J S^{2}}{a} R\left[\ln \left(\frac{2 R}{a}\right)-1\right] \propto A R\left[\ln \left(\frac{2 R}{a}\right)-1\right]
$$

For the Fe-rich Fe-Zr metallic glasses, the cluster sizes deduced from magnetic-viscosity measurements [31] are in the range of $R=3$ to $10 \mathrm{~nm}$, while $a \approx 0.25 \mathrm{~nm}$. Consequently, $2 R / a$ takes values in the range of 24 to 80 , giving a positive value for $\Delta E$. The energy barrier for the cluster varies almost linearly with $R$ in the interested $R$ range since the logarithmic term changes little in the same $R$ range. To conclude this section, we determined the energy barrier for a particle of radius $R$ in the curling mode to be proportional to $\Delta E \propto A R$, which replaces the $\Delta E=K V$ term valid for homogeneous rotation.

\section{With coupling}

The coupling between the clusters and the infinite (FM) matrix is treated in the framework of a mean-field model. The magnetic field, $H$, is replaced by $H+\lambda M$ in Eq. (A2), where $\lambda$ is the coupling constant,

$$
E_{\mathrm{tot}}=A \frac{\sin ^{2} \omega}{R^{2}}+(H+\lambda M) M_{s} \cos \omega .
$$


The matrix is assumed to be an ideal FM having $M=M_{s}$ for $H>0$ and $M=-M_{s}$ for $H<0$, where the saturation magnetization, $M_{s}$, of the clusters is equal with that of the FM matrix. A similar calculation as above gives for the energy barrier of a particle of radius $R$ without field,

$$
\Delta E=A R\left(1-\frac{R^{2} \lambda M_{s}^{2}}{2 A}\right)^{2}
$$

The physical picture behind this mean-field model is that the thermal energy $(k T)$ is aided by the mean-field of the FM matrix to facilitate the magnetization reversal of the clusters. The blocking temperature, $T_{B}$, is determined by the condition
$\Delta E=25 k T_{B}$

$$
\begin{aligned}
T_{B} & =\frac{A R}{25 k}\left(1-\frac{R^{2} \lambda M_{s}^{2}}{2 A}\right)^{2}=\frac{A R}{25 k}\left(1-\frac{m_{s} \lambda M_{s}}{2 A R}\right)^{2} \\
& =\frac{\Delta E^{0}}{25 k}\left(1-\frac{E_{c}}{2 \Delta E^{0}}\right)^{2},
\end{aligned}
$$

where the cluster moment $m_{s}=R^{3} M_{s}$ was introduced in the second expression and the notations $\Delta E^{0}=A R$ and $E_{c}=$ $m_{s} \lambda M_{s}$ were applied in the third expression. Here $\Delta E^{0}$ is the energy barrier for curling without coupling, and $E_{c}$ is the coupling energy. Equation (3) has a physical meaning only if $E_{c} /\left(2 \Delta E^{0}\right) \leqslant 1$, which is fulfilled (see the Discussion).
[1] H. Hiroyoshi and K. Fukamichi, Phys. Lett. A 85, 242 (1981).

[2] K. Fukamichi, Amorphous Metallic Alloys, edited by F. E. Luborsky (Butterworth, London, 1983), p. 317.

[3] K. Fukamichi, T. Goto, H. Komatsu, and H. Wakabayashi, Proc. $4^{\text {th }}$ Int. Conf. on Phys. Magn. Mater., edited by W. Gorzkowski, H. K. Lahowicz, and H. Symczak (World Scientific, Singapore, 1989), p. 354.

[4] D. A. Read, T. Moyo, and G. C. Hallam, J. Magn. Magn. Mater. 44, 279 (1984).

[5] D. H. Ryan, J. M. D. Coey, E. Batalla, Z. Altounian, and J. O. Ström-Olsen, Phys. Rev. B 35, 8630 (1987).

[6] H. Ren and D. H. Ryan, Phys. Rev. B 51, 15885 (1995).

[7] W. Kümmerle and U. Gradmann, Solid State Commun. 24, 33 (1977).

[8] G. Xiao and C. L. Chien, Phys. Rev. B 35, 8763 (1987).

[9] S. Krompiewski, U. Krauss, and U. Krey, Physica B 161, 219 (1989).

[10] N. P. Kovalenko, Yu. P. Krasny, and U. Krey, Physics of Amorphous Metals (Wiley-VCH, Berlin, 2001), p. 171.

[11] D. H. Ryan, J. O. Ström-Olsen, R. Provencher, and M. Townsend, J. Appl. Phys. 64, 5787 (1988).

[12] M. Gabay and G. Toulouse, Phys. Rev. Lett. 47, 201 (1981).

[13] N. Saito, H. Hiroyoshi, K. Fukamichi, and Y. Nakagawa, J. Phys. F: Metal Phys. 16, 911 (1986).

[14] D. A. Read, T. Moyo, and G. C. Hallam, J. Magn. Magn. Mater. 54-57, 309 (1986).

[15] D. A. Read, G. C. Hallam, and M. Chirwa, J. Magn. Magn. Mater. 82, 83 (1989).

[16] T. Moyo, J. Magn. Magn. Mater. 154, 201 (1996).

[17] S. N. Kaul, J. Phys.: Condens. Matter 3, 4027 (1991).

[18] S. N. Kaul and V. Siruguri, J. Phys.: Condens. Matter 4, 505 (1992)

[19] S. N. Kaul and Ch. V. Mohan, J. Appl. Phys. 71, 6090 (1992).

[20] S. N. Kaul and Ch. V. Mohan, J. Appl. Phys. 71, 6103 (1992).

[21] T. Moyo, J. Phys.: Condens. Matter 8, 8915 (1996).

[22] L. Karlsson, R. L. McGreevy, and J. D. Wicks, J. Phys.: Condens. Matter 11, 9249 (1999).

[23] R. Lorenz and J. Hafner, J. Magn. Magn. Mater. 139, 209 (1995).

[24] M. Yu and Y. Kakehashi, J. Magn. Magn. Mater. 162, 189 (1996).

[25] M. Ghafari, N. Chmielek, W. Keune, and C. P. Foley, Hyperfine Interact. 54, 527 (1990).
[26] I. Vincze, D. Kaptás, T. Kemény, L. F. Kiss, and J. Balogh, Phys. Rev. Lett. 73, 496 (1994); 73, 1735 (1994) (erratum).

[27] I. Bakonyi, J. Magn. Magn. Mater. 324, 3961 (2012).

[28] I. Bakonyi, Acta Mater. 53, 2509 (2005).

[29] D. Kaptás, T. Kemény, L. F. Kiss, L. Gránásy, J. Balogh, and I. Vincze, J. Non-Cryst. Solids 156-158, 336 (1993).

[30] L. F. Kiss, T. Kemény, I. Vincze, and L. Gránásy, J. Magn. Magn. Mater. 135, 161 (1994).

[31] L. F. Kiss, T. Kemény, and I. Vincze, J. Phys.: Condens. Matter 9, 10501 (1997).

[32] A. R. Wildes, N. Cowlam, S. Al-Heniti, L. F. Kiss, and T. Kemény, Physica B 276-278, 712 (2000).

[33] A. R. Wildes, N. Cowlam, S. Al-Heniti, L. F. Kiss, and T. Kemény, J. Magn. Magn. Mater. 226-230, 1470 (2001).

[34] A. R. Wildes, J. R. Stewart, N. Cowlam, S. Al-Heniti, L. F. Kiss, and T. Kemény, J. Phys.: Condens. Matter 15, 675 (2003).

[35] K. Shirakawa, K. Fukamichi, T. Kaneko, and T. Masumoto, Physica B 119, 192 (1983).

[36] K. Shirakawa, K. Fukamichi, T. Kaneko, and T. Masumoto, Sci. Rep. RITU A 31, 54 (1983).

[37] J. Kamarád and Z. Arnold, Physica B 139-140, 382 (1986).

[38] A. Zamani, A. Hallén, P. Nordblad, G. Andersson, B. Hjövarsson, and P. E. Jönsson, J. Magn. Magn. Mater. 346, 138 (2013).

[39] A. Zamani, R. Moubah, M. Ahlberg, H. Stopfel, U. B. Arnalds, A. Hallén, B. Hjövarsson, G. Andersson, and P. E. Jönsson, J. Appl. Phys. 117, 143903 (2015).

[40] R. Moubah, A. Zamani, A. Olsson, S. Shi, A. Hallén, S. Carlson, D. Arvanitis, P. Nordblad, B. Hjövarsson, and P. Jönsson, Appl. Phys. Express 6, 053001 (2013).

[41] R. Moubah, M. Ahlberg, A. Zamani, A. Olsson, S. Shi, Z. Sun, S. Carlson, A. Hallén, B. Hjövarsson, and P. E. Jönsson, J. Appl. Phys. 116, 053906 (2014).

[42] D. Mishra, M. Gurram, A. Reddy, A. Perumal, P. Saravanan, and A. Srinivasan, Mater. Sci. Eng. B 175, 253 (2010).

[43] H. Fujimori, K. Nakanishi, K. Shirakawa, T. Masumoto, T. Kaneko, and N. S. Kazama, Proc. 4th Int. Conf. on Rapidly Quenched Metals, Vol. II, edited by K. Suzuki and T. Masumoto (The Japan Inst. Metals, Sendai, 1982), p. 1629.

[44] K. Fukamichi, H. Hiroyoshi, T. Kaneko, T. Masumoto, and K. Shirakawa, J. Appl. Phys. 53, 8107 (1982). 
[45] K. Fukamichi, K. Shirakawa, T. Kaneko, and T. Masumoto, Proc. 5th Int. Conf. on Rapidly Quenched Metals, edited by S. Steeb and H. Warlimont (North-Holland, Amsterdam, 1985), p. 1165.

[46] K. Fukamichi, K. Shirakawa, Y. Satoh, T. Masumoto, and T. Kaneko, J. Magn. Magn. Mater. 54-57, 231 (1986).

[47] K. Fukamichi, H. Hiroyoshi, K. Shirakawa, T. Masumoto, and T. Kaneko, IEEE Trans. Magn. 22, 424 (1986).

[48] T. Goto, C. Murayama, N. Mori, H. Wakabayashi, K. Fukamichi, and H. Komatsu, J. de Physique 49, C8-1143 (1988).

[49] K. Fukamichi, K. Aoki, T. Masumoto, T. Goto, C. Murayama, and N. Mori, J. Alloys and Compounds 256, 18 (1997).

[50] I. Bakonyi, F. Mehner, M. Rapp, Á. Cziráki, H. Kronmüller, and R. Kirchheim, Z. Metallkd. 86, 619 (1995).

[51] J. Kamarád, Z. Machátová, and Z. Arnold, Rev. Sci. Instrum. 75, 5022 (2004).

[52] H.-P. Liermann, Z. Konôpková, W. Morgenroth, K. Glazyrin, J. Bednarčík, E. E. McBride, S. Petitgirard, J.T. Delitz, M. Wendt, Y. Bican, A. Ehnes, I. Schwark, A. Rothkirch, M. Tischer, J. Heuer, H. Schulte-Schrepping, T. Kracht, and H. Franz, J. Synchr. Rad. 22, 908 (2015).

[53] H. K. Mao, J. Xu, and P. M. Bell, J. Geophys. Res. 91, 4673 (1986).

[54] A. P. Hammersley, S. O. Svensson, M. Hanfland, A. N. Fitch, and D. Häusermann, High Press. Res. 14, 235 (1996).

[55] J. F. Wang, R. Li, R. J. Xiao, T. Xu, Y. Li, Z. Q. Liu, L. Huang, N. B. Hua, G. Li, Y. C. Li, and T. Zhang, Appl. Phys. Lett. 99, 151911 (2011).

[56] P. W. Bridgman, The Physics of High Pressure (Bells and Sons, London, 1958).

[57] L. F. Kiss, D. Kaptás, and N. Hegman, in Magnetic Hysteresis in Novel Magnetic Materials, NATO ASI Series, Series E: Applied Sciences, Vol. 338, edited by G. Hadjipanayis (Kluwer Academic Publishers, London, 1997), p. 755.

[58] K. Balakrishnan, P. D. Babu, V. Ganesan, R. Srinivasan, and S. N. Kaul, J. Magn. Magn. Mater. 250, 110 (2002).

[59] T. Kaneko, K. Shirakawa, S. Ohnuma, M. Nose, H. Fujimori, and T. Masumoto, J. Appl. Phys. 52, 1826 (1981).

[60] K. Shirakawa, T. Kaneko, M. Nose, S. Ohnuma, H. Fujimori, and T. Masumoto, J. Appl. Phys. 52, 1829 (1981).

[61] H. Tange, K. Inoue, and K. Shirakawa, J. Magn. Magn. Mater. 54-57, 303 (1986).
[62] J. Kamarád, J. Schneider, and Z. Arnold, Phys. Stat. Sol. (a) 67, K85 (1981).

[63] H. Tange, T. Matsuyama, A. Chikazawa, K. Konishi, and T. Kamimori, J. Magn. Magn. Mater. 177-181, 125 (1998).

[64] I. Bakonyi, V. Skumryev, R. Reisser, G. Hilscher, L. K. Varga, L. F. Kiss, H. Kronmüller, and R. Kirchheim, Z. Metallkd. 88, 117 (1997).

[65] H. Tange, K. Inoue, and K. Shirakawa, J. Magn. Magn. Mater. 68, 102 (1987).

[66] L. F. Kiss, T. Kemény, J. Bednarčík, J. Kamarád, Z. Arnold, Z. Konôpková, and H.-P. Liermann, J. Phys.: Condens. Matter 25, 346002 (2013).

[67] L. F. Kiss and N. Hegman, J. Magn. Magn. Mater. 140-144, 293 (1995).

[68] B. D. Cullity and C. D. Graham, Introduction to Magnetic Materials (John Wiley and Sons, Hoboken, 2009), p. 383.

[69] ibid. as Ref. [68], p. 368.

[70] E. H. Frei, S. Shtrikman, and D. Treves, Phys. Rev. 106, 446 (1957).

[71] A. Aharoni, J. Appl. Phys. 30, 70S (1959).

[72] ibid. as Ref. [68], p. 133.

[73] ibid. as Ref. [68], p. 282.

[74] S. N. Kaul and P. D. Babu, J. Phys.: Condens. Matter 10, 1563 (1998).

[75] R. Krishnan, K. V. Rao, and H. H. Liebermann, J. Appl. Phys. 55, 1823 (1984).

[76] P. L. Paulose and V. Nagarajan, Phys. Rev. B 54, 14934 (1996).

[77] R. Pauthenet, J. Appl. Phys. 53, 2029 (1982).

[78] ibid. as Ref. [68], p. 388.

[79] D. Wagner and E. P. Wohlfarth, J. Phys. F 11, 2417 (1981).

[80] D. Wagner and E. P. Wohlfarth, J. Phys. F 9, 717 (1979).

[81] E. P. Wohlfarth, Physica B 91, 305 (1977).

[82] W. X. Zhang and W. L. Zhang, J. Appl. Phys. 117, 163917 (2015).

[83] ibid. as Ref. [68], p. 369.

[84] A. H. Morrish, The Physical Principles of Magnetism (John Wiley and Sons, London, 1965), p. 340.

[85] F. Sommer, T. Tarnóczi, K. Russew, Á. Cziráki, L. F. Kiss, L. K. Varga, and I. Bakonyi, Z. Metallkd. 89, 256 (1998).

[86] M. Jurczyk, B. Szymanski, A. Wrzeciono, and A. J. Janicki, Physica Status Solidi (a) 74, K69 (1982).

[87] S. N. Kaul, Phys. Rev. B 27, 6923 (1983). 